\title{
ADAPTIVE PARAMETER ESTIMATION OF HYPERBOLIC DISTRIBUTED PARAMETER SYSTEMS: NON-SYMMETRIC DAMPING AND SLOWLY TIME VARYING SYSTEMS
}

\author{
H.T. BANKS AND M.A. DEMETRIOU
}

\begin{abstract}
In this paper a model reference-based adaptive parameter estimator for a wide class of hyperbolic distributed parameter systems is considered. The proposed state and parameter estimator can handle hyperbolic systems in which the damping sesquilinear form may not be symmetric (or even present) and a modification to the standard adaptive law is introduced to account for this lack of symmetry (or absence) in the damping form. In addition, the proposed scheme is modified for systems in which the input operator, bounded or unbounded, is also unknown. Parameters that are slowly time varying are also considered in this scheme via an extension of finite dimensional results. Using a Lyapunov type argument, state convergence is established and with the additional assumption of persistence of excitation, parameter convergence is shown. An approximation theory necessary for numerical implementation is established and numerical results are presented to demonstrate the applicability of the above parameter estimators.
\end{abstract}

\section{INTRODUCTION}

The adaptive parameter estimation of second order distributed parameter systems was initially studied by Demetriou and Rosen, [15], written in a second order setting and by Scondo [25], Demetriou [14] and Baumeister et al. [12] written as a first order system having strong damping. The scheme presented there did not account for systems with non-symmetric damping bilinear forms. Such a system, which motivated the current work, was observed in the adaptive estimation of structural acoustic models [4] where lack of symmetry in the damping form was observed and thus the scheme presented in [15] was no longer applicable. A modification to the adaptive scheme was therefore required in order to account for the lack of such a symmetry.

In this note we propose various modifications to account for this (lack of symmetry) and therefore rendering the scheme implementable. In addition,

H.T. Banks: Center for Research in Scientific Computation, North Carolina State University, Raleigh, NC 27695-8205, USA. E-mail: htbanks@eos.ncsu.edu.

M.A. Demetriou: Department of Mechanical Engineering, Worcester Polytechnic Institute, Worcester, MA 01609, USA. E-mail: mdemetri@kimon.wpi.edu.

Research supported in part by the Air Force Office of Scientific Research under grant AFOSR F49620-95-1-0236 and in part by NASA under grant NAG-1-1600.

Received by the journal December 7, 1996. Revised November 6, 1997. Accepted for publication February 19, 1998.

(C) Société de Mathématiques Appliquées et Industrielles. Typeset by $\mathrm{LATEX}_{\mathrm{E}}$ 
if it is known that the parameters are (slowly) time varying, then a state and parameter estimator is proposed to estimate these time varying parameters. Specifically, if it is known that the (unknown) parameters converge to a steady state value, then with the additional assumption of persistence of excitation, the scheme can guarantee that the parameter estimates converge to the steady state value of the system's parameters. Another issue that arises in many hyperbolic distributed parameter systems is when the system has no damping at all. While the "no-damping" case does not occur physically, it is often the model used for systems with very light and/or poorly understood damping. This case is also treated here, and the persistence of excitation condition, required for parameter convergence, is modified in order to account for the absence of the damping term. It is worth mentioning that even though the system under consideration does not have a damping term, the proposed estimator requires one in order to guarantee convergence. Furthermore, this estimator appears to be more general than the one presented in [14] (systems with strong damping) or in [25] (systems with no damping), because the estimator operators here are not simply chosen as the plant's operators evaluated at some optimal parameter, but rather some other general and not necessarily parameterized operators. In all cases above, unlike [15] and [25], the parameters in the input operator are assumed to be unknown and therefore the adaptive schemes can identify these parameters in the input operator. In addition, these schemes can handle the case where the input operator is unbounded. A similar result was established in [14] for the case with symmetric damping form. The issue of unknown parameters in the source term is of great importance in the control of flexible structures and especially when control is implemented via smart actuators. In this case, parameters related to the geometric and physical properties of these smart actuators are usually known to within a percentage of the actual value and often vary (slowly) with time. These fluctuations exhibit destabilizing effects in controlling these structures with the end result of poor, if not inadequate, control performance.

The following section introduces the underlying spaces that are needed to analyze the system and its estimator and gives conditions imposed on the system that are required for parameter convergence. This essentially defines the class of systems for which the adaptive parameter estimation schemes are applicable. Section 3 provides a summary of the stability and convergence arguments for such an estimator. The adaptive parameter estimation scheme for systems with (slowly) time varying parameters is presented in Section 4. Since the proposed state and parameter estimators are infinite dimensional, an approximation theory, which will be used for implementation purposes, is developed in Section 5. Section 6, which includes examples and numerical simulations of the structural acoustic system [4], is added to demonstrate the applicability of the above theoretical results while Section 7 summarizes the results and concludes with further open problems in the area of adaptive parameter identification of distributed parameter systems. 


\section{The Plant and the Estimator}

Let $H$ be a real Hilbert space with inner product $\langle\cdot, \cdot\rangle$ and corresponding induced norm $|\cdot|$, and let $V_{1}$ and $V_{2}$ be real reflexive Banach spaces with norms denoted by $\|\cdot\|_{V_{1}}$ and $\|\cdot\|_{V_{2}}$, respectively. We assume that $V_{1}$ is embedded densely and continuously in $V_{2}$ and that $V_{2}$ is embedded densely and continuously in $H$. It then follows that (see, for example, $[6,10]$ )

$$
V_{1} \hookrightarrow V_{2} \hookrightarrow H \cong H^{*} \hookrightarrow V_{2}^{*} \hookrightarrow V_{1}^{*},
$$

where $H^{*}, V_{2}^{*}$, and $V_{1}^{*}$ denote the conjugate duals of $H, V_{2}$, and $V_{1}$, respectively. Since the embeddings in (2.1) are dense and continuous, $[21,26,27$, $28]$, we assume that there exist positive constants, $K_{V_{2}}, K_{V_{1}}$, and $K_{V_{2}, V_{1}}$,

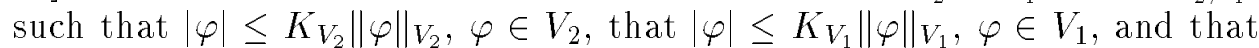
$\|\varphi\|_{V_{2}} \leq K_{V_{2}, V_{1}}\|\varphi\|_{V_{1}}, \varphi \in V_{1}$. We denote the usual operator norms on $V_{2}^{*}$ and $V_{1}^{*}$ by $\|\cdot\|_{V_{2}^{*}}$ and $\|\cdot\|_{V_{1}^{*}}$, respectively. The duality pairing, denoted by $\langle\cdot, \cdot\rangle_{V_{1}^{*}, V_{1}}$, is the extension by continuity of the inner product $\langle\cdot, \cdot\rangle$ from $H \times V_{1}$ to $V_{1}^{*} \times V_{1}$; hence, elements $\varphi^{*} \in V_{1}^{*}$ have the representation $\varphi^{*}(\varphi)=\left\langle\varphi^{*}, \varphi\right\rangle_{V_{1}^{*}, V_{1}}$, see $[7,10]$. As it was pointed out in $[4,6,7,15], V_{2}$ can either be $V_{1}, H$ or some intermediate space, depending on the damping form chosen.

Let $Q$ be a real Hilbert space (henceforth the parameter space) with inner product $\langle\cdot, \cdot\rangle_{Q}$ and corresponding induced norm $|\cdot|_{Q}$, and for each $q \in Q$ let $\sigma_{1}(q ; \cdot, \cdot): V_{1} \times V_{1} \rightarrow \mathbb{R}^{1}$ be a bilinear form on $V_{1}$ satisfying

(A1) (Symmetry) $\sigma_{1}(q ; \varphi, \psi)=\sigma_{1}(q ; \psi, \varphi), \quad \varphi, \psi \in V_{1}$, and $q \in Q$,

for at least one tuning parameter $q^{*} \in Q$, we have

(A2) (Boundedness)

$$
\left|\sigma_{1}\left(q^{*} ; \varphi, \psi\right)\right| \leq \alpha^{0}\left(q^{*}\right)\|\varphi\|_{V_{1}}\|\psi\|_{V_{1}}, \quad \varphi, \psi \in V_{1}, \text { with } \alpha^{0}\left(q^{*}\right)>0,
$$

(A3) (Coercivity)

$$
\sigma_{1}\left(q^{*} ; \varphi, \varphi\right) \geq \alpha_{0}\left(q^{*}\right)\|\varphi\|_{V_{1}}^{2}, \quad \varphi \in V_{1}, \text { with } \alpha_{0}\left(q^{*}\right)>0,
$$

(A4) (Linearity) the map $q \rightarrow \sigma_{1}(q ; \varphi, \psi)$ from $Q$ into $\mathbb{R}^{1}$ is linear for each $\varphi, \psi \in V_{1}$.

Similarly, for each $q \in Q$, let $\sigma_{2}(q ; \cdot, \cdot): V_{2} \times V_{2} \rightarrow \mathbb{R}^{1}$ be a bilinear form on $V_{2}$ satisfying

(B1) (Symmetry) $\sigma_{2}(q ; \varphi, \psi)=\sigma_{2}(q ; \psi, \varphi), \quad \varphi, \psi \in V_{2}$,

(B2) (Boundedness)

$$
\left|\sigma_{2}\left(q^{*} ; \varphi, \psi\right)\right| \leq \beta^{0}\left(q^{*}\right)\|\varphi\|_{V_{2}}\|\psi\|_{V_{2}}, \quad \varphi, \psi \in V_{2},
$$

(B3) (Coercivity) $\sigma_{2}\left(q^{*} ; \varphi, \varphi\right) \geq \beta_{0}\left(q^{*}\right)\|\varphi\|_{V_{2}}^{2}, \quad \varphi \in V_{2}$,

(B4) (Linearity) The map $q \rightarrow \sigma_{2}(q ; \varphi, \psi)$ from $Q$ into $\mathbb{R}^{1}$ is linear for each $\varphi, \psi \in V_{2}$,

where $\beta^{0}\left(q^{*}\right), \beta_{0}\left(q^{*}\right)>0$, and $q^{*}$ is the same fixed element of $Q$ appearing in Assumptions $(A 2)$ and $(A 3)$ above.

For each $q \in Q$, let $b(q ; \cdot, \cdot): H \times V_{1} \rightarrow \mathbb{R}^{1}$ be a bilinear form satisfying (C1) (Boundedness)

$$
|b(q ; \vartheta, \psi)| \leq\left.|q|_{Q}|\vartheta||| \psi\right|_{V_{1}}, \quad \vartheta \in H, \quad \psi \in V_{1},
$$


$(C 2)$ (Linearity) The map $q \rightarrow b(q ; \vartheta, \psi)$ from $Q$ into $\mathbb{R}^{1}$ is linear for $\vartheta \in H$ and $\psi \in V_{1}$.

For $q \in Q$ define the linear operators $A_{1}(q)$ and $A_{2}(q)$ from $V_{1}$ into $V_{1}^{*}$ and $V_{2}$ into $V_{2}^{*}$ by

$$
\begin{array}{ll}
\left\langle A_{1}(q) \varphi, \psi\right\rangle=\sigma_{1}(q ; \varphi, \psi), & \varphi, \psi \in V_{1}, \\
\left\langle A_{2}(q) \varphi, \psi\right\rangle=\sigma_{2}(q ; \varphi, \psi), & \varphi, \psi \in V_{2} .
\end{array}
$$

In addition, we define the input operator $B(q): H \rightarrow V_{1}^{*}$ by

$$
\langle B(q) \vartheta, \psi\rangle_{V_{1}^{*}, V}=b(q ; \vartheta, \psi), \quad \vartheta \in H, \psi \in V_{1} .
$$

For $q \in Q$ we consider the second order linear initial value problem given in variational form by

$$
\begin{gathered}
\left\langle z_{t t}(t), \varphi\right\rangle_{V_{1}^{*}, V_{1}}+\sigma_{2}\left(q ; z_{t}(t), \varphi\right)+\sigma_{1}(q ; z(t), \varphi)=b(q ; f(t), \varphi), \\
\varphi \in V_{1}, t>0 \\
z(0)=z_{0}, \quad z_{t}(0)=z_{1},
\end{gathered}
$$

where $z_{t}$ denotes time differentiation, $z_{0} \in V_{1}, z_{1} \in H$, and $f \in L_{2}(0, \infty ; H)$.

The well posedness of the system (2.2), (2.3) with symmetric $\sigma_{2}$ was established in [6] using linear semigroup theory. The case where $\sigma_{2}$ is not symmetric was treated in $[5,8]$ for structural acoustic interaction models.

The identification objective is to design a state and parameter estimator using the plant state $\left(z(t), z_{t}(t)\right)$ and the plant input $f(t)$ in order to identify the unknown plant parameter $q$ adaptively.

We now make the assumption of an admissible plant as it was defined in [15], namely a boundedness condition on the state of the system.

Definition 2.1. An admissible plant is a triple $(q, z, f)$ with $q \in Q$ and $\left(z, z_{t}\right)$ a solution to the initial value problem $(2.2),(2.3)$ corresponding to $q$ and $f$, for which there exists constants $\lambda>0$ such that

$$
\left|\sigma_{2}\left(p ; z_{t}(t), \varphi\right)+\sigma_{1}(p ; z(t), \varphi)-b(p ; f(t), \varphi)\right| \leq \lambda|p|_{Q}\|\varphi\|_{V_{2}},
$$

for almost every $t>0, p \in Q$ and $\varphi \in V_{1}$.

Following the results in [14], it is possible to specify sufficient conditions for a solution $\left(z, z_{t}\right)$ to the initial value problem $(2.2),(2.3)$ to have the necessary regularity for $(q, z, f)$ to be an admissible plant.

\subsection{Non-Symmetric Damping}

Given an admissible plant $(q, z, f)$ and a tuning parameter $q^{*} \in Q$, we define in the same manner the estimator for $q$ and $z$, denoted by $\hat{q}$ and $\hat{z}$ respectively, in the form of the initial value problem

$$
\begin{gathered}
\left\langle\hat{z}_{t t}(t), \varphi\right\rangle+\sigma_{2}\left(q^{*} ; \hat{z}_{t}(t), \varphi\right)+\sigma_{1}\left(q^{*} ; \hat{z}(t), \varphi\right)+\sigma_{2}\left(\hat{q}(t) ; z_{t}(t), \varphi\right) \\
+\sigma_{1}(\hat{q}(t) ; z(t), \varphi) \\
=b(\hat{q}(t) ; f(t), \varphi)+\sigma_{2}\left(q^{*} ; z_{t}(t), \varphi\right)+\sigma_{1}\left(q^{*} ; z(t), \varphi\right) \\
\varphi \in V_{1}, t>0,
\end{gathered}
$$




$$
\begin{gathered}
\left\langle\hat{q}_{t}(t), p\right\rangle_{Q}+\sigma_{2}\left(p ; z_{t}(t), z(t)-\hat{z}(t)\right)+\sigma_{1}(p ; z(t), z(t)-\hat{z}(t)) \\
-b(p ; f(t), z(t)-\hat{z}(t))+\gamma \sigma_{2}\left(p ; z_{t}(t), z_{t}(t)-\hat{z}_{t}(t)\right) \\
+\gamma \sigma_{1}\left(p ; z(t), z_{t}(t)-\hat{z}_{t}(t)\right)-\gamma b\left(p ; f(t), z_{t}(t)-\hat{z}_{t}(t)\right)=0, \\
\quad p \in Q, t>0, \\
\hat{z}(0) \in V_{1}, \quad \hat{z}_{t}(0) \in H, \quad \hat{q}(0) \in Q .
\end{gathered}
$$

REMARK 2.2. In the case that condition $(B 1)$ is not satisfied, i.e. $\sigma_{2}(q ; \cdot, \cdot)$ is not symmetric, the above adaptive estimator does not satisfy the conditions presented in [15] and therefore it cannot be implemented. We propose two ways to alleviate such a problem.

$\left(S_{1}\right)$ We can impose the condition that the bilinear form satisfies the symmetry condition $(B 1)$ only when it is evaluated at the tuning parameter $q^{*}$, namely

$$
\sigma_{2}\left(q^{*} ; \varphi, \psi\right)=\sigma_{2}\left(q^{*} ; \psi, \varphi\right), \quad \forall \varphi, \psi \in V_{1} \text { and some } q^{*} \in Q .
$$

$\left(S_{2}\right)$ Replace the non-symmetric term $\sigma_{2}\left(q^{*} ; \varphi, \psi\right)$ in $(2.4)$ with the symmetric term

$$
\frac{1}{2}\left[\sigma_{2}\left(q^{*} ; \varphi, \psi\right)+\sigma_{2}\left(q^{*} ; \psi, \varphi\right)\right], \quad \varphi, \psi \in V_{1} .
$$

The first option seems more attractive for implementational purposes, but it might happen that equation (2.7) can never be satisfied for any tuning parameter $q^{*} \in Q$ that simultaneously satisfies $\left(S_{1}\right),(A 2),(A 3),(B 2)$ and (B3). Of course, if there exists such a $q^{*}$, then $\left(S_{1}\right)$ is the preferred modification. If, on the other hand, there does not exist a $q^{*} \in Q$ such that $\left(S_{1}\right)$ is satisfied, then $\left(S_{2}\right)$ must be implemented. With the second option taken, equation (2.4) then becomes

$$
\begin{aligned}
\left\langle\hat{z}_{t t}(t), \varphi\right\rangle+\frac{1}{2}\left[\sigma_{2}\left(q^{*} ; \hat{z}_{t}(t), \varphi\right)+\sigma_{2}\left(q^{*} ; \varphi, \hat{z}_{t}(t)\right)\right] & \\
& +\sigma_{1}\left(q^{*} ; \hat{z}(t), \varphi\right)+\sigma_{2}\left(\hat{q}(t) ; z_{t}(t), \varphi\right)+\sigma_{1}(\hat{q}(t) ; z(t), \varphi) \\
= & b(\hat{q}(t) ; f(t), \varphi)+\frac{1}{2}\left[\sigma_{2}\left(q^{*} ; z_{t}(t), \varphi\right)+\sigma_{2}\left(q^{*} ; \varphi, z_{t}(t)\right)\right] \\
& +\sigma_{1}\left(q^{*} ; z(t), \varphi\right), \quad \varphi \in V_{1}, t>0,
\end{aligned}
$$

We note that the parameters $q^{*} \in Q$ and $\gamma>0$ can be thought of as gains, or tuning parameters, which are used to "tune" the estimator, see [15]. Of course $q^{*}$ must be such that Assumptions $(A 2),(A 3),(B 2)$, and $(B 3)$ (and $\left(S_{1}\right)$ when applicable) are satisfied. We note as well, that weighting the $Q$ inner product can also serve to tune the scheme, see $[15,16]$.

A third option seems to be the most general and will be the one treated throughout these pages. Instead of searching for an optimal parameter $q^{*} \in Q$ such that Assumptions $(A 2)-(A 3),(B 2)-(B 3)$ and $\left(S_{1}\right)$ or $\left(S_{2}\right)$ are satisfied, one can introduce bilinear forms $\Sigma_{i}(\cdot, \cdot): V_{i} \times V_{i} \rightarrow \mathbb{R}^{1}$ that do not depend on any parameter $q \in Q$ or necessarily bear any resemblance to the bilinear forms $\sigma_{i}(q ; \cdot, \cdot)$, and that satisfy Assumptions $(A 1)-(A 3)$ and (B1)-(B3) with known bounds. That is, let $\Sigma_{1}(\cdot, \cdot): V_{1} \times V_{1} \rightarrow \mathbb{R}^{1}$ be a bilinear form on $V_{1}$ satisfying

(Ā1) (Symmetry) $\Sigma_{1}(\varphi, \psi)=\Sigma_{1}(\underline{\psi}, \varphi), \quad \varphi, \psi \in \underline{V_{1}}$,

$(\bar{A} 2)$ (Boundedness) $\left|\Sigma_{1}(\varphi, \psi)\right| \leq \overline{\alpha^{0}}\|\varphi\|_{V_{1}}\|\psi\|_{V_{1}}, \quad \overline{\alpha^{0}}>0, \quad \varphi, \psi \in V_{1}$, 
(Ä3) (Coercivity) $\Sigma_{1}(\varphi, \varphi) \geq \overline{\alpha_{0}}\|\varphi\|_{V_{1}}^{2}, \quad \overline{\alpha_{0}}>0, \quad \varphi \in V_{1}$, and let $\Sigma_{2}(\cdot, \cdot): V_{2} \times V_{2} \rightarrow \mathbb{R}^{1}$ be a bilinear form on $V_{2}$ satisfying

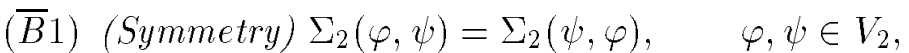
$(\bar{B} 2)$ (Boundedness) $\left|\Sigma_{2}(\varphi, \psi)\right| \leq \overline{\beta^{0}}\|\varphi\|_{V_{2}}\|\psi\|_{V_{2}}, \quad \overline{\beta^{0}}>0, \quad \varphi, \psi \in V_{2}$, $(\bar{B} 3)$ (Coercivity) $\Sigma_{2}(\varphi, \varphi) \geq \overline{\beta_{0}}\|\varphi\|_{V_{2}}^{2}, \quad \overline{\beta_{0}}>0, \quad \varphi \in V_{2}$.

This can also be viewed as being a more general case of the adaptive scheme presented in $[15,25]$ where $\sigma_{i}\left(q^{*} ; \cdot, \cdot\right)$ is a specific form of $\Sigma_{i}(\cdot, \cdot), i=1,2$. Then, the state and parameter estimator equivalents of $(2.4)-(2.6)$ are now given by

$$
\begin{gathered}
\left\langle\hat{z}_{t t}(t), \varphi\right\rangle+\Sigma_{2}\left(\hat{z}_{t}(t), \varphi\right)+\Sigma_{1}(\hat{z}(t), \varphi)+\sigma_{2}\left(\hat{q}(t) ; z_{t}(t), \varphi\right)+\sigma_{1}(\hat{q}(t) ; z(t), \varphi) \\
=b(\hat{q}(t) ; f(t), \varphi)+\Sigma_{2}\left(z_{t}(t), \varphi\right)+\Sigma_{1}(z(t), \varphi), \varphi \in V_{1}, t>0, \\
\left\langle\hat{q}_{t}(t), p\right\rangle_{Q}+\sigma_{2}\left(p ; z_{t}(t), z(t)-\hat{z}(t)\right)+\sigma_{1}(p ; z(t), z(t)-\hat{z}(t)) \\
-b(p ; f(t), z(t)-\hat{z}(t))+\gamma \sigma_{2}\left(p ; z_{t}(t), z_{t}(t)-\hat{z}_{t}(t)\right) \\
+\gamma \sigma_{1}\left(p ; z(t), z_{t}(t)-\hat{z}_{t}(t)\right)-b\left(p ; f(t), z_{t}(t)-\hat{z}_{t}(t)\right)=0, \\
p \in Q, t>0, \\
\hat{z}(0) \in V_{1}, \quad \hat{z}_{t}(0) \in H, \quad \hat{q}(0) \in Q .10
\end{gathered}
$$

Assumptions ( $A 4)$ and (B4) imply that the system (2.9), (2.10) is linear in $(\hat{z}, \hat{q})$. One way that the initial value problem $(2.9),(2.10)$ and $(2.11)$ can be shown to be well posed is in the sense of the existence of a unique mild or generalized solution. This can be argued via the theory of infinite dimensional evolution equations as presented in, for example, Pazy [23], Tanabe [27] or the more relevant treatment by Demetriou and Rosen [15] and Scondo [25] for the symmetric or absent $\sigma_{2}$ case. We duplicate, for sake of completeness, the procedure used in [15] and present the required modifications.

Let $\left\{X,\langle\cdot, \cdot\rangle_{X}\right\}$ be the Hilbert space defined by $X=V_{1} \times H$ and introducing an additional tuning parameter $\gamma$, we define the $X$-inner product

$$
\langle\varphi, \psi\rangle_{X}=\gamma\left\{\Sigma_{1}\left(\varphi_{1}, \psi_{1}\right)+\left\langle\varphi_{2}, \psi_{2}\right\rangle\right\}+\left\langle\varphi_{1}, \psi_{2}\right\rangle+\left\langle\psi_{1}, \varphi_{2}\right\rangle+\Sigma_{2}\left(\varphi_{1}, \psi_{1}\right),
$$

for $\varphi=\left(\varphi_{1}, \varphi_{2}\right), \psi=\left(\psi_{1}, \psi_{2}\right) \in X, \gamma \in \mathbb{R}$. It can easily be shown [15] that if $\gamma>0$ is sufficiently large, then $\langle\cdot, \cdot\rangle_{X}$ is in fact an inner product on $X$ and moreover, that the corresponding induced norm, $|\cdot|_{X}$, is equivalent to the norm on $X$ induced by the more standard inner product on $X$ given by

$$
(\varphi, \psi)_{X}=\Sigma_{1}\left(\varphi_{1}, \psi_{1}\right)+\left\langle\varphi_{2}, \psi_{2}\right\rangle
$$

for $\varphi=\left(\varphi_{1}, \varphi_{2}\right), \psi=\left(\psi_{1}, \psi_{2}\right) \in X$. In fact, these arguments will be given in the proofs of Lemma 3.1 and convergence results in the next section (see also [15]). Now let $Y=V_{1} \times V_{1}$ be endowed with the norm

$$
\|\varphi\|_{Y}=\left\{\left\|\varphi_{1}\right\|_{V_{1}}^{2}+\left\|\varphi_{2}\right\|_{V_{1}}^{2}\right\}^{\frac{1}{2}}
$$

for $\varphi=\left(\varphi_{1}, \varphi_{2}\right) \in Y$. Then $Y$ is a reflexive Banach space and $Y \hookrightarrow X \hookrightarrow Y^{*}$ with the embeddings dense and continuous.

We rewrite $(2.9),(2.10)$ in first order form as

$$
\frac{d}{d t}\left[\begin{array}{c}
x(t) \\
\hat{q}(t)
\end{array}\right]=\left[\begin{array}{cc}
\mathcal{A} & \mathcal{B}(t) \\
-\mathcal{B}(t)^{*} & 0
\end{array}\right]\left[\begin{array}{c}
x(t) \\
\hat{q}(t)
\end{array}\right]+\mathcal{F}(t), \quad t>0,
$$


where $\mathcal{A} \in \mathcal{L}\left(Y, Y^{*}\right)$ and $\mathcal{B}(t) \in \mathcal{L}\left(Q, Y^{*}\right)$, are given by

$$
\mathcal{A} \varphi=\left[\begin{array}{cc}
0 & I \\
-\mathcal{A}_{1} & -\mathcal{A}_{2}
\end{array}\right]\left[\begin{array}{l}
\varphi_{1} \\
\varphi_{2}
\end{array}\right], \quad \varphi=\left(\varphi_{1}, \varphi_{2}\right) \in Y
$$

and

$$
\mathcal{B}(t) q=\left[\begin{array}{c}
0 \\
-A_{2}(q) z_{t}(t)-A_{1}(q) z(t)+B(q) f(t)
\end{array}\right], \quad q \in Q
$$

respectively (the operators $A_{1}(q)$ and $A_{2}(q)$ are as they have been defined above, $\mathcal{A}_{1} \in \mathcal{L}\left(V_{1}, V_{1}^{*}\right)$ and $\mathcal{A}_{2} \in \mathcal{L}\left(V_{2}, V_{2}^{*}\right)$ are the operators that correspond to the bilinear forms $\Sigma_{1}, \Sigma_{2}$ and are defined similar to the operators $A_{1}(q), A_{2}(q), \mathcal{B}(t)^{*} \in \mathcal{L}(Y, Q)$ is the Banach space adjoint of $\mathcal{B}(t)$ (that is, recalling that above embeddings, that $\left\langle\mathcal{B}(t)^{*} \varphi, q\right\rangle_{Q}=\langle\varphi, \mathcal{B}(t) q\rangle_{X}, \varphi \in Y$, $q \in Q$ ), and the forcing term is

$$
\mathcal{F}(t)=\left[\begin{array}{c}
0 \\
\mathcal{A}_{2} z_{t}(t)+\mathcal{A}_{1} z(t) \\
\mathcal{B}(t)^{*}\left(z(t), z_{t}(t)\right)
\end{array}\right], \quad t>0
$$

Since $(q, z, f)$ is assumed to be an admissible plant, then $\mathcal{F} \in L_{2}(0, T ;(Y \times$ $Q)^{*}$ ) for all $T>0$. We assume that $z$ is such that $A_{2}(q) z_{t}(t)+A_{1}(q) z(t) \in H$, $t \geq 0, q \in Q$, and that $\mathcal{A}_{2} z_{t}(t)+\mathcal{A}_{1} z(t) \in H, t \geq 0$. In addition we assume that the map $t \rightarrow A_{2}(q) z_{t}(t)+A_{1}(q) z(t)$, for each $q \in Q$, is strongly continuously differentiable in $H$. Let $\mathcal{X}=X \times Q$, let the domain $\mathcal{D}$ be

$$
\mathcal{D}=\left\{(\varphi, q) \in \mathcal{X}: \varphi=\left(\varphi_{1}, \varphi_{2}\right) \in Y \text { and } \mathcal{A}_{1} \varphi_{1}+\mathcal{A}_{2} \varphi_{2} \in H\right\}
$$

and define the family of operators $\{\tilde{\mathcal{A}}(t)\}_{t \geq 0}, \tilde{\mathcal{A}}(t): \mathcal{D} \subset \mathcal{X} \rightarrow \mathcal{X}$ by

$$
\tilde{\mathcal{A}}(t)=\left[\begin{array}{cc}
\mathcal{A} & \mathcal{B}(t) \\
-\mathcal{B}(t)^{*} & 0
\end{array}\right], \quad t \geq 0 .
$$

Using the results in [15] it can be shown that $\{\tilde{\mathcal{A}}(t)\}_{t \geq 0}$ is a stable family of infinitesimal generators of $\mathcal{C}_{0}$ semigroups on $\mathcal{X}$ and that the map $t \rightarrow$ $\tilde{\mathcal{A}}(t)(\varphi, q)$ for $(\varphi, q) \in \mathcal{D}$ is strongly continuously differentiable in $\mathcal{X}$. It then follows, [23], that the system $(2.9),(2.10),(2.11)$ admits a unique mild solution. When the initial data, $(x(0), \hat{q}(0))$, is sufficiently regular (i.e. $(x(0), \hat{q}(0)) \in \mathcal{D}$ ) and $\mathcal{F}$ is sufficiently regular (which essentially depends upon the regularity of the plant state, $z$ ) we have a strong solution to the initial value problem $(2.9)-(2.11)$. In the rest of this note, we assume that the initial data and the plant are sufficiently regular to ensure that the initial value problem $(2.9)-(2.11)$ has a strong solution.

Let $e(t):=\hat{z}(t)-z(t)$ denote the state error and $r(t):=\hat{q}(t)-q$ the parameter error, with $(q, z, f)$ a plant and $(\hat{q}, \hat{z})$ a solution to the initial value problem $(2.9)-(2.11)$. In the next section we will show that under no additional assumptions we have state error convergence and that under the additional assumption of persistence of excitation can guarantee parameter convergence. 


\subsection{Systems with no Damping}

In this subsection we consider systems with no damping term, given in weak form by

$$
\begin{gathered}
\left\langle z_{t t}(t), \varphi\right\rangle_{V_{1}^{*}, V_{1}}+\sigma_{1}(q ; z(t), \varphi)=b(q ; f(t), \varphi), \quad \varphi \in V_{1}, t>0, \\
z(0)=z_{0}, \quad z_{t}(0)=z_{1},
\end{gathered}
$$

where $z_{0} \in V_{1}, z_{1} \in H$, and $f \in L_{2}(0, \infty ; H)$. In this case we only utilize a Gelfand triple with $V_{1} \hookrightarrow H \simeq H^{*} \hookrightarrow V_{1}^{*}$, [28].

The well posedness of the system $(2.16),(2.17)$ with bounded input operator can easily be established using linear semigroup theory, see [10, 20, 21, $23,26,28]$. The case of unbounded input operator is treated in [7]. Once again, it is possible to specify sufficient conditions for a $\operatorname{solution}\left(z, z_{t}\right)$ to $(2.16),(2.17)$ to have the necessary regularity for $(q, z, f)$ to be an admissible plant.

The corresponding definition for an admissible plant, in this case, is the triple $(q, z, f)$ with $\left(z, z_{t}\right)$ a solution to $(2.16)$, for which there exist $\lambda>0$ such that

$$
\left|\sigma_{1}(p ; z(t), \varphi)-b(p ; f(t), \varphi)\right| \leq \lambda|p|_{Q}|| \varphi \|_{V_{2}}
$$

for almost every $t>0, p \in Q$ and $\varphi \in V_{1}$. In the proposed state and parameter estimator below, we utilize a damping sesquilinear form $\sigma_{2}$ even though $\sigma_{2}$ does not occur naturally in the system $(2.16),(2.17)$. The motivation behind this is to enhance the convergence properties of the state error (both $\|e(t)\|_{V_{1}}$ and $\left.\left|e_{t}(t)\right|\right)$ to zero; this will become clearer in the ensuing stability analysis. In this case the proposed state and parameter estimators take the form

$$
\begin{gathered}
\left\langle\hat{z}_{t t}(t), \varphi\right\rangle+\sigma_{2}\left(q^{*} ; \hat{z}_{t}(t), \varphi\right)+\sigma_{1}\left(q^{*} ; \hat{z}(t), \varphi\right)+\sigma_{1}(\hat{q}(t) ; z(t), \varphi) \\
=b(\hat{q}(t) ; f(t), \varphi)+\sigma_{2}\left(q^{*} ; z_{t}(t), \varphi\right)+\sigma_{1}\left(q^{*} ; z(t), \varphi\right), \varphi \in V, t>0 \\
\left\langle q_{t}(t), p\right\rangle_{Q}+\sigma_{1}(p ; z(t), z(t)-\hat{z}(t))-b(p ; f(t), z(t)-\hat{z}(t)) \\
+\gamma\left[\sigma_{1}\left(p ; z(t), z_{t}(t)-\hat{z}_{t}(t)\right)-b\left(p ; f(t), z_{t}(t)-\hat{z}_{t}(t)\right)\right]=0 \\
\quad p \in Q, t>0, \\
\hat{z}(0) \in V_{1}, \quad \hat{z}_{t}(0) \in H, \quad \hat{q}(0) \in Q,
\end{gathered}
$$

where $\sigma_{2}$ (a design term) is chosen to satisfy Assumptions (A1) - (A4). We note, however, that by employing a 5-space setting as in $(2.1), \sigma_{2}$ could alternatively be chosen to satisfy Assumptions $(B 1)$ - $(B 4)$ which permits one to obtain the desired results under, in general, much weaker assumptions on $\sigma_{2}$. The reader is also directed to [25] for a similar treatment of second order systems with no damping in a 3 -space setting where that author chooses $\sigma_{2}\left(q^{*} ; \cdot, \cdot\right)$ of the estimator to be the same as $\sigma_{1}\left(q^{*} ; \cdot, \cdot\right)$ of the system.

As in the case of subsection 2.1, we define the space $X=V_{1} \times H$ with the same inner product

$\langle\varphi, \psi\rangle_{X}=\gamma\left\{\sigma_{1}\left(q^{*} ; \varphi_{1}, \psi_{1}\right)+\left\langle\varphi_{2}, \psi_{2}\right\rangle\right\}+\left\langle\varphi_{1}, \psi_{2}\right\rangle+\left\langle\psi_{1}, \varphi_{2}\right\rangle+\sigma_{2}\left(q^{*} ; \varphi_{1}, \psi_{1}\right)$, for $\varphi=\left(\varphi_{1}, \varphi_{2}\right), \psi=\left(\psi_{1}, \psi_{2}\right) \in X$, where $q^{*} \in Q$ is as it was defined in Assumptions (A2), (A3) (or equivalently in Assumptions (B2), (B3)).

ESAIM: COCV, MAY 1998, VOL. 3, 133-162 
REMARK 2.3. In the no damping case the bilinear form $\sigma_{2}$, proposed in (2.19), (2.20), is chosen to satisfy all four conditions $(A 1)-(A 4)$, and this of course results in a Gelfand triple setting rather than a Gelfand quintuple setting. The symmetry condition is also imposed even when $\sigma_{2}(q ; \cdot, \cdot)$ is not evaluated at the tuning parameter $q^{*}$. This can be done since $\sigma_{2}$ is a purely design term. One can further modify the above estimator by using the results of the third option (general option) to replace $\sigma_{1}\left(q^{*} ; \cdot, \cdot\right)$ by $\Sigma_{1}(\cdot, \cdot)$ and $\sigma_{2}\left(q^{*} ; \cdot, \cdot\right)$ by $\Sigma_{2}(\cdot, \cdot)$ if more design flexibility is desired, at the expense of increased complexity via a Gelfand quintuple. This then becomes a more general case that includes the treatment of symmetric damping in $[12,15]$ and of no damping in [25].

Similarly, we define the reflexive Banach space $Y=V_{1} \times V_{1}$, endowed with the norm

$$
\|\varphi\|_{Y}=\left\{\left\|\varphi_{1}\right\|_{V_{1}}^{2}+\left\|\varphi_{2}\right\|_{V_{1}}^{2}\right\}^{\frac{1}{2}}
$$

for $\left(\varphi_{1}, \varphi_{2}\right) \in Y$ with $Y \hookrightarrow H \hookrightarrow Y^{*}$ and the embeddings dense and contin110us. We rewrite (2.19)-(2.21) in first order form as

$$
\frac{d}{d t}\left[\begin{array}{l}
x(t) \\
\hat{q}(t)
\end{array}\right]=\left[\begin{array}{cc}
\mathcal{A} & \mathcal{B}(t) \\
-\mathcal{B}(t)^{*} & 0
\end{array}\right]\left[\begin{array}{l}
x(t) \\
\hat{q}(t)
\end{array}\right]+\mathcal{F}(t), \quad t>0,
$$

where in this case $\mathcal{A} \in \mathcal{L}\left(Y, Y^{*}\right)$ and $\mathcal{B}(t) \in \mathcal{L}\left(Q, Y^{*}\right)$, are now given by

$$
\mathcal{A} \varphi=\left[\begin{array}{cc}
0 & I \\
-A_{1}\left(q^{*}\right) & -A_{2}\left(q^{*}\right)
\end{array}\right]\left[\begin{array}{l}
\varphi_{1} \\
\varphi_{2}
\end{array}\right], \quad \varphi=\left(\varphi_{1}, \varphi_{2}\right) \in Y,
$$

and

$$
\mathcal{B}(t) q=\left[\begin{array}{c}
0 \\
-A_{1}(q) z(t)+B(q) f(t)
\end{array}\right], \quad q \in Q,
$$

respectively (once again the operators $A_{1}(q)$ and $A_{2}(q)$ are as they have been defined in subsection 2.1 with $A_{2}(q)$ now in $\left.\mathcal{L}\left(V_{1}, V^{*}\right)\right), \mathcal{B}(t)^{*} \in \mathcal{L}(Y, Q)$ is again the Banach space adjoint of $\mathcal{B}(t)$, and

$$
\mathcal{F}(t)=\left[\begin{array}{c}
0 \\
A_{2}\left(q^{*}\right) z_{t}(t)+A_{1}\left(q^{*}\right) z(t) \\
\mathcal{B}(t)^{*}\left(z(t), z_{t}(t)\right)
\end{array}\right], \quad t>0 .
$$

From the equivalent definition of the plant, equation (2.18), we have $\mathcal{F} \in$ $L_{2}\left(0, T ; Y^{*}\right)$ for all $T>0$. Using similar arguments as in Section 2 , we can show the well posedness of the state and parameter estimator, (2.19)-(2.21).

Note that if the general case is used (Remark 2.3) then the $\mathcal{A}$ operator is now given by $(2.13)$ and $\mathcal{F}(t)$ by $(2.15)$

\section{Stability And Convergence}

We now establish the convergence of the state estimator

$$
\lim _{t \rightarrow \infty}\|e(t)\|_{V_{1}}=0 \text { and } \lim _{t \rightarrow \infty}\left|e_{t}(t)\right|=0
$$

and, with the additional assumption of persistence of excitation, parameter convergence. That is, $\lim _{t \rightarrow \infty}|r(t)|_{Q}=\lim _{t \rightarrow \infty}|\hat{q}(t)-q|_{Q}=0$. We assume throughout this section that $(q, z, f)$ is an admissible plant (see Definition 2.1). 


\subsection{Non-Symmetric Damping}

Using the plant equation (2.2) with non-symmetric $\sigma_{2}$ (i.e. (B1) not satisfied), the state estimator (2.9), and the parameter estimator (2.10), we have that $e$ and $r$ satisfy the linear, homogeneous, non-autonomous, initial value problem given in weak or variational form by

$$
\begin{aligned}
& \left\langle e_{t t}(t), \varphi\right\rangle+\Sigma_{2}\left(e_{t}(t), \varphi\right)+\Sigma_{1}(e(t), \varphi)+\sigma_{2}\left(r(t) ; z_{t}(t), \varphi\right)+\sigma_{1}(r(t) ; z(t), \varphi) \\
& =b(r(t) ; f(t), \varphi), \quad \varphi \in V_{1}, t>0, \\
& \left\langle r_{t}(t), p\right\rangle_{Q}-\sigma_{2}\left(p ; z_{t}(t), e(t)\right)-\sigma_{1}(p ; z(t), e(t))+b(p ; f(t), e(t)) \\
& -\gamma \sigma_{2}\left(p ; z_{t}(t), e_{t}(t)\right)-\gamma \sigma_{1}\left(p ; z(t), e_{t}(t)\right)+\gamma b\left(p ; f(t), e_{t}(t)\right)=0, \\
& p \in Q, t>0, \\
& e(0) \in V_{1}, \quad e_{t}(0) \in H, \quad r(0) \in Q .
\end{aligned}
$$

We next establish a Lyapunov-like estimate for the system (3.1) - (3.3). In essence, it is shown that the derivative of an energy function is non-positive along the trajectories of the error equations (3.1), (3.2).

LEMMA 3.1. ([15]) If the tuning parameter $\gamma$ satisfies

$$
\gamma>\max \left\{K_{V_{1}}, \frac{K_{V_{1}}}{\overline{\alpha_{0}}}, \frac{K_{V_{2}}^{2}}{\overline{\beta_{0}}}\right\}
$$

then there exist constants $\rho, k>0$ such that for all $t>0$

$$
\|e(t)\|_{V_{1}}^{2}+\left|e_{t}(t)\right|^{2}+|r(t)|_{Q}^{2}+\rho \int_{0}^{t}\left\{\|e(s)\|_{V_{1}}^{2}+\left\|e_{s}(s)\right\|_{V_{2}}^{2}\right\} d s \leq \xi,
$$

where $\xi=k\left\{\|e(0)\|_{V_{1}}^{2}+\left|e_{t}(0)\right|^{2}+|r(0)|_{Q}^{2}\right\}$.

Proof. Following the treatment in [15], we define the energy functional, $E$ : $[0, \infty) \rightarrow \mathbb{R}^{1}$, for the system $(3.1),(3.2)$ by

$$
\begin{aligned}
E(t)= & \gamma\left\{\Sigma_{1}(e(t), e(t))+\left|e_{t}(t)\right|^{2}\right\}+2\left\langle e(t), e_{t}(t)\right\rangle \\
& +\Sigma_{2}(e(t), e(t))+|r(t)|_{Q}^{2} \\
= & \left|\left(\begin{array}{c}
e(t) \\
e_{t}(t)
\end{array}\right)\right|_{X}^{2}+|r(t)|_{Q}^{2} .
\end{aligned}
$$

Then, using $(3.1),(3.2)$, and assumptions $(\bar{A} 3)$ and $(\bar{B} 3)$, we obtain

$$
\begin{aligned}
E_{t}(t)= & \gamma\left\{2 \Sigma_{1}\left(e(t), e_{t}(t)\right)+2\left\langle e_{t t}(t), e_{t}(t)\right\rangle\right\}+2\left\langle e_{t}(t), e_{t}(t)\right\rangle \\
& +2\left\langle e(t), e_{t t}(t)\right\rangle+2 \Sigma_{2}\left(e_{t}(t), e(t)\right)+2\left\langle r_{t}(t), r(t)\right\rangle_{Q} \\
= & -2 \gamma \Sigma_{2}\left(e_{t}(t), e_{t}(t)\right)-2 \Sigma_{1}(e(t), e(t))+2\left|e_{t}(t)\right|^{2} \\
\leq & -2 \overline{\alpha_{0}}\|e(t)\|_{V_{1}}^{2}-\left\{2 \gamma \overline{\beta_{0}}-2 K_{V_{2}}^{2}\right\}\left\|e_{t}(t)\right\|_{V_{2}}^{2} \\
\leq & -\rho_{0}\left\{\|e(t)\|_{V_{1}}^{2}+\left\|e_{t}(t)\right\|_{V_{2}}^{2}\right\},
\end{aligned}
$$

for some $\rho_{0}=\min \left\{2 \overline{\alpha_{0}}, 2 \gamma \overline{\beta_{0}}-2 K_{V_{2}}^{2}\right\}>0$, since, by assumption, $\gamma>$ $K_{V_{2}}^{2} / \overline{\beta_{0}}$. Consequently,

$$
E(t)+\rho_{0} \int_{0}^{t}\left\{\|e(s)\|_{V_{1}}^{2}+\left\|e_{s}(s)\right\|_{V_{2}}^{2}\right\} d s \leq E(0) .
$$


From assumptions $(\bar{A} 2)$ and $(\bar{B} 2)$, see also [15], we find that

$$
E(t) \leq \kappa_{R}\left\{\|e(t)\|_{V_{1}}^{2}+\left|e_{t}(t)\right|^{2}+|r(t)|_{Q}^{2}\right\},
$$

for some $\kappa_{R}>0$ and from (3.5) that

$$
\kappa_{L}\left\{\|e(t)\|_{V_{1}}^{2}+\left|e_{t}(t)\right|^{2}+|r(t)|_{Q}^{2}\right\} \leq E(t),
$$

for some $\kappa_{L}>0$. The desired result then follows from (3.7), (3.8), (3.9), and the assumed lower bound on $\gamma$.

The convergence of the state estimate is established below without imposing any additional assumptions. Let $(q, z, f)$ be an admissible plant. If $\gamma>\max \left\{K_{V_{1}}, K_{V_{1}} / \overline{\alpha_{0}}, K_{V_{2}}^{2} / \overline{\beta_{0}}\right\}$, then the energy functional $E$ given by (3.5) is non-increasing,

$$
\lim _{t \rightarrow \infty}\|e(t)\|_{V_{1}}=0 \text { and } \lim _{t \rightarrow \infty}\left|e_{t}(t)\right|=0 .
$$

The proof of the state convergence is similar to the one given in [15] for the symmetric case and is given in greater detail in Appendix A of the companion report [3].

REMARK 3.2. ([15]) In the case that $V_{1}=V_{2}$, inspection of the definition of $E$ given in (3.5) reveals that Lemma 3.1 and the error convergence (3.10) can be proved with the somewhat weaker assumption on $\gamma$ that

$$
\gamma>\max \left\{K_{V_{1}}, \frac{K_{V_{1}}-\overline{\beta_{0}}}{\overline{\alpha_{0}}}, \frac{K_{V_{2}}^{2}}{\overline{\beta_{0}}}\right\} .
$$

In order to establish parameter convergence we require the notion of persistence of excitation.

Definition 3.3. A plant $(q, z, f)$ is said to be persistently excited via the input $f$, if there exists $T_{0}, \delta_{0}, \epsilon_{0}>0$ such that for each $p \in Q$ with $|p|_{Q}=1$ and each $t_{1}>0$ sufficiently large, there exists a $\tilde{t} \in\left[t_{1}, t_{1}+T_{0}\right]$ such that

$$
\left\|\int_{\tilde{t}}^{\tilde{t}+\delta_{0}}\left\langle A_{2}(p) z_{\tau}(\tau), \cdot\right\rangle+\left\langle A_{1}(p) z(\tau), \cdot\right\rangle-\langle B(p)(\tau), \cdot\rangle d \tau\right\|_{V_{1}^{*}} \geq \epsilon_{0} .
$$

With the above condition assumed, we have that if the plant $(q, z, f)$ is persistently excited then

$$
\lim _{t \rightarrow \infty}|r(t)|_{Q}=0
$$

The proof of parameter convergence follows from two technical lemmas which are stated and proven in Appendix A of [3]. It is almost identical to the symmetric case presented in [15].

REMARK 3.4. Continuing with possible extensions for the non-symmetric case as was presented in Remark 2.2, we also present a fourth possible way which is less complicated but changes the bound on the constant $\gamma$. If the original state estimator (with no modifications) given by (2.4) is used, then 
in the proof of Lemma 3.1 we have that

$$
\begin{aligned}
E_{t}(t) \leq & -2 \alpha_{0}\left(q^{*}\right)\|e(t)\|_{V_{1}}^{2}-\left\{2 \gamma \beta_{0}\left(q^{*}\right)-2 K_{V_{2}}^{2}\right\}\|e(t)\|_{V_{1}}^{2} \\
& +\sigma_{2}\left(q^{*} ; e_{t}(t), e(t)\right)-\sigma_{2}\left(q^{*} ; e(t), e_{t}(t)\right) \\
\leq & -\left\{2 \alpha_{0}\left(q^{*}\right)-\beta^{0}\left(q^{*}\right)\right\}\|e(t)\|_{V_{1}}^{2} \\
& -\left\{2 \gamma \beta_{0}\left(q^{*}\right)-2 K_{V_{2}}^{2}-\beta^{0}\left(q^{*}\right) K_{V_{2}, V_{1}}^{2}\right\}\|e(t)\|_{V_{1}}^{2},
\end{aligned}
$$

which imposes the additional condition $2 \alpha_{0}\left(q^{*}\right)-\beta^{0}\left(q^{*}\right)>0$ and decreases the lower bound of $\gamma$ to $\gamma \geq \frac{2 K_{V_{2}}^{2}-\beta^{0}\left(q^{*}\right) K_{V_{2}, V_{1}}^{-2}}{2 \beta_{0}\left(q^{*}\right)}$. These changes will affect the choice of the constant $c_{2}$ and $\rho_{0}$ in the proof of parameter convergence, (see Appendix A of [3]) which will eventually affect the rate of convergence $\gamma_{0}$ of $E(t)$ to zero, see [16]. Concluding about this option, either the level of excitation, $\epsilon_{0}$ must increase or the length of the time window $\delta_{0}$ for Definition 3.3 must decrease.

\subsection{Systems With NO Damping}

Assuming that the purely designed term $\sigma_{2}$ is used (Remark 2.3) then the resulting state and parameter error equations become

$$
\begin{gathered}
\left\langle e_{t t}(t), \varphi\right\rangle+\sigma_{2}\left(q^{*} ; e_{t}(t), \varphi\right)+\sigma_{1}\left(q^{*} ; \epsilon(t), \varphi\right)+\sigma_{1}(r(t) ; z(t), \varphi) \\
=b(r(t) ; f(t), \varphi), \quad \varphi \in V_{1}, t>0, \\
\left\langle r_{t}(t), p\right\rangle_{Q}-\sigma_{1}(p ; z(t), \epsilon(t))+b(p ; f(t), e(t)) \\
-\gamma\left\{\sigma_{1}\left(p ; z(t), e_{t}(t)\right)-b\left(p ; f(t), e_{t}(t)\right)\right\}=0, \quad p \in Q, t>0, \\
e(0) \in V_{1}, \quad e_{t}(0) \in H, \quad r(0) \in Q .
\end{gathered}
$$

The persistence of excitation condition reduces to requiring that for $T_{0}$, $\delta_{0}, \epsilon_{0}>0$, for each $p \in Q$ with $|p|_{Q}=1$ and each $t_{1}>$ sufficiently large, there exists a $\tilde{t} \in\left[t_{1}, t_{1}+T\right]$ such that

$$
\left\|\int_{\tilde{t}}^{\tilde{t}+\delta_{0}}\left\langle A_{1}(p) z(\tau), \cdot\right\rangle-\langle B(p) f(\tau), \cdot\rangle d \tau\right\|_{V_{1}^{*}} \geq \epsilon_{0} .
$$

The corresponding result in state error convergence is then summarized. Let $(q, z, f)$ be an admissible plant. If $\gamma>\max \left\{K_{V_{1}}, K_{V_{1}} / \alpha_{0}\left(q^{*}\right), K_{V_{1}}^{2} / \alpha_{0}\left(q^{*}\right)\right\}$, then the energy functional $E$ given by (3.5) is non-increasing,

$$
\lim _{t \rightarrow \infty}\|e(t)\|_{V_{1}}=0 \text { and } \lim _{t \rightarrow \infty}\left|e_{t}(t)\right|=0 .
$$

The arguments leading to the proof of the state error convergence are similar to the case with damping presented in Appendix A of [3] and are therefore omitted. The differences are in the definition of the (admissible) plant given by (2.18).

Once again, in order to achieve parameter convergence we impose the persistence of excitation condition given by (3.14). If the condition of persistence of excitation, given by (3.14), is satisfied, then

$$
\lim _{t \rightarrow \infty}|r(t)|_{Q}=0 \text {. }
$$


The proof of parameter convergence once again resembles the arguments used in Appendix A of [3] for the case with damping. The differences are in the choice of the constant $\mu$ used in the proof of Lemma A.1 and in the choice of $c_{1}, c_{2}, \gamma_{0}$ and $g(t)$ used in the proof of state error convergence with damping.

REMARK 3.5. Following Remark 2.3, if the general option with a 5-space setting is used, then the parameter $\gamma$ required to guarantee convergence is given by (3.4).

\section{Slowly Time-VARying Systems}

We now turn our attention to systems with time varying parameters. Specifically, we treat systems whose parameters are assumed to asymptotically converge to an (unknown) steady state value. Consider the system (with a symmetric $\sigma_{2}(q ; \cdot, \cdot)$ assumed here for simplicity, i.e. satisfying Assumption $(B 1))$

$$
\begin{gathered}
\left\langle z_{t t}(t), \varphi\right\rangle_{V_{1}^{*}, V_{1}}+\sigma_{2}\left(q(t) ; z_{t}(t), \varphi\right)+\sigma_{1}(q(t) ; z(t), \varphi)=b(q(t) ; f(t), \varphi), \\
\varphi \in V_{1}, t>0, \\
z(0)=z_{0} \in V_{1}, \quad z_{t}(0)=z_{1} \in H, \quad q(0)=q_{0} \in Q,
\end{gathered}
$$

where $z_{0} \in V_{1}, z_{1} \in H, f \in L_{2}(0, \infty ; H)$, and $q(0) \in Q$ is some unknown initial condition of the parameter $q(t)$. We assume that the parameter $q(t)$ satisfies

$$
\left\langle q_{t}(t), p\right\rangle_{Q}=\left\langle A_{q} q(t), p\right\rangle_{Q}+\left\langle c_{s s}, p\right\rangle_{Q},
$$

where $q(0) \in Q, A_{q}$ is the infinitesimal generator of an exponentially stable $\mathcal{C}_{0}$ semigroup on $Q$ satisfying

$$
\left\langle A_{q} p, p\right\rangle_{Q} \leq-\kappa_{q}|p|_{Q}^{2}, \quad p \in Q, \quad \text { some } \kappa_{q}>0,
$$

and $c_{s s}$ is related to the steady state value of $q(t)$. Following the results in $[1,2]$ for finite dimensional adaptive systems and in [23] for $Q$ being an infinite dimensional parameter space with $q(t)$ now denoting the mild solution, we have that (4.3) satisfies

$$
\lim _{t \rightarrow \infty} q(t)=q_{s s}=-A_{q}^{-1} c_{s s}
$$

where $q_{s s}$ is the steady state value of $q(t)$. It is assumed in this case that both $q(0)$ and $q_{s s}$ (equivalently $c_{s s}$ ) are unknown. The only parameter assumed known here is the dissipation bound $\kappa_{q}$.

REMARK 4.1. We can also assume that $c_{s s}$ is a function of time and that there exists a steady state value for $c_{s s}(t)$ (assumed bounded and measurable on $[0, \infty)$ ), namely, $c_{s s}^{\infty}=\lim _{t \rightarrow \infty} c_{s s}(t)$, so that $q_{s s}=-A_{q}^{-1} c_{s s}^{\infty}$, see $[18,23]$.

The definition of an admissible plant will be taken to be the same presented in Section 2, namely Definition 2.1. We can follow the procedure given in [20], [23], or [28] to establish the well posedness of the system (4.1) $-(4.3)$. 
We now proceed to establish a state and parameter estimator that will guarantee the convergence of the state error to zero, and by imposing the additional condition of persistence of excitation, parameter convergence. Following the treatment of finite dimensional results for slowly time varying parameters, see for example [22], we re-introduce the parameter error given by $\tilde{q}(t):=\hat{q}(t)-q_{s s}$, instead of the more conventional term, $\hat{q}(t)-q(t)$, and define $\bar{q}(t):=q(t)-q_{s s}$. The equations for the state and parameter estimator are the same as the ones given for hyperbolic systems with symmetric damping and time invariant parameters by (2.4) - (2.6), namely

$$
\begin{aligned}
& \left\langle\hat{z}_{t t}(t), \varphi\right\rangle+\sigma_{2}\left(q^{*} ; \hat{z}_{t}(t), \varphi\right)+\sigma_{1}\left(q^{*} ; \hat{z}(t), \varphi\right)+\sigma_{2}\left(\hat{q}(t) ; z_{t}(t), \varphi\right) \\
& +\sigma_{1}(\hat{q}(t) ; z(t), \varphi) \\
& =\quad b(\hat{q}(t) ; f(t), \varphi)+\sigma_{2}\left(q^{*} ; z_{t}(t), \varphi\right)+\sigma_{1}\left(q^{*} ; z(t), \varphi\right), \varphi \in V, t>0,(4.5) \\
& \left\langle\hat{q}_{t}(t), p\right\rangle_{Q}+\sigma_{2}\left(p ; z_{t}(t), z(t)-\hat{z}(t)\right)+\sigma_{1}(p ; z(t), z(t)-\hat{z}(t)) \\
& -b(p ; f(t), z(t)-\hat{z}(t))+\gamma \sigma_{2}\left(p ; z_{t}(t), z_{t}(t)-\hat{z}_{t}(t)\right) \\
& +\gamma \sigma_{1}\left(p ; z(t), z_{t}(t)-\hat{z}_{t}(t)\right)-\gamma b\left(p ; f(t), z_{t}(t)-\hat{z}_{t}(t)\right)=0, \\
& p \in Q, t>0, \\
& \hat{z}(0) \in V_{1}, \quad \hat{z}_{t}(0) \in H, \quad \hat{q}(0) \in Q .
\end{aligned}
$$

Following the procedure given in Section 2, we duplicate the steps taken for establishing the well posedness of the state and parameter estimator, $(4.5)$ - (4.7). We first assume that Assumptions (A1) - (A4), (B1) - (B4) are satisfied for $q, q^{*} \in Q$. Let $\left\{X,\langle\cdot, \cdot\rangle_{X}\right\}$ be the Hilbert space defined by $X=V_{1} \times H$ and now define

$\langle\varphi, \psi\rangle_{X}=\gamma\left\{\sigma_{1}\left(q^{*} ; \varphi_{1}, \psi_{1}\right)+\left\langle\varphi_{2}, \psi_{2}\right\rangle\right\}+\left\langle\varphi_{1}, \psi_{2}\right\rangle+\left\langle\psi_{1}, \varphi_{2}\right\rangle+\sigma_{2}\left(q^{*} ; \varphi_{1}, \psi_{1}\right)$, for $\varphi=\left(\varphi_{1}, \varphi_{2}\right), \psi=\left(\psi_{1}, \psi_{2}\right) \in X$, where $q^{*} \in Q$ is as it was defined in Assumptions $(A 2),(A 3),(B 2)$, and (B3).

We rewrite $(4.5),(4.6)$ in first order form as

$$
\frac{d}{d t}\left[\begin{array}{l}
x(t) \\
\hat{q}(t)
\end{array}\right]=\left[\begin{array}{cc}
\mathcal{A} & \mathcal{B}(t) \\
-\mathcal{B}(t)^{*} & 0
\end{array}\right]\left[\begin{array}{c}
x(t) \\
\hat{q}(t)
\end{array}\right]+\mathcal{F}(t), \quad t>0,
$$

where $\mathcal{A} \in \mathcal{L}\left(Y, Y^{*}\right)$ and $\mathcal{B}(t) \in \mathcal{L}\left(Q, Y^{*}\right)$, are now given by

$$
\mathcal{A} \varphi=\left[\begin{array}{cc}
0 & I \\
-A_{1}\left(q^{*}\right) & -A_{2}\left(q^{*}\right)
\end{array}\right]\left[\begin{array}{l}
\varphi_{1} \\
\varphi_{2}
\end{array}\right], \quad \varphi=\left(\varphi_{1}, \varphi_{2}\right) \in Y,
$$

and

$$
\mathcal{B}(t) q=\left[\begin{array}{c}
0 \\
-A_{2}(q) z_{t}(t)-A_{1}(q) z(t)+B(q) f(t)
\end{array}\right], \quad q \in Q
$$

respectively, $\mathcal{B}(t)^{*} \in \mathcal{L}(Y, Q)$, and $\mathcal{F}(t)$ is now given by

$$
\mathcal{F}(t)=\left[\begin{array}{c}
0 \\
A_{2}\left(q^{*}\right) z_{t}(t)+A_{1}\left(q^{*}\right) z(t) \\
\mathcal{B}(t)^{*}\left(z(t), z_{t}(t)\right)
\end{array}\right], \quad t>0 .
$$


The rest of the arguments are similar to the ones used in Section 2 and are therefore omitted. The resulting error equations are now given by

$$
\begin{gathered}
\left\langle e_{t t}(t), \varphi\right\rangle+\sigma_{2}\left(q^{*} ; e_{t}(t), \varphi\right)+\sigma_{1}\left(q^{*} ; e(t), \varphi\right)+\sigma_{2}\left(\tilde{q}(t) ; z_{t}(t), \varphi\right) \\
+\sigma_{1}(\tilde{q}(t) ; z(t), \varphi)-\sigma_{2}\left(\bar{q}(t) ; z_{t}(t), \varphi\right)-\sigma_{1}(\bar{q}(t) ; z(t), \varphi) \\
=b(\tilde{q}(t) ; f(t), \varphi)-b(\bar{q}(t) ; f(t), \varphi), \quad \varphi \in V, t>0 \\
\left\langle\tilde{q}_{t}(t), p\right\rangle_{Q}-\sigma_{2}\left(p ; z_{t}(t), e(t)\right)-\sigma_{1}(p ; z(t), e(t))+b(p ; f(t), e(t)) \\
-\gamma \sigma_{2}\left(p ; z_{t}(t), e_{t}(t)\right)-\gamma \sigma_{1}\left(p ; z(t), e_{t}(t)\right)+\gamma b\left(p ; f(t), e_{t}(t)\right)=0 \\
p \\
\left\langle\bar{q}_{t}(t), p\right\rangle, t>0 \\
=\left\langle\frac{d}{d t} q(t)-\frac{d}{d t} q_{s s}, p\right\rangle=\left\langle A_{q} q(t), p\right\rangle+\left\langle c_{s s}, p\right\rangle \\
=\left\langle A_{q}\left[q(t)-q_{s s}+q_{s s}\right], p\right\rangle+\left\langle c_{s s}, p\right\rangle \\
=\left\langle A_{q} \bar{q}(t), p\right\rangle+\left\langle A_{q} q_{s s}+c_{s s}, p\right\rangle=\left\langle A_{q} \bar{q}(t), p\right\rangle \\
e(0) \in V_{1}, \quad e_{t}(0) \in H, \quad \tilde{q}(0), \bar{q}(0) \in Q
\end{gathered}
$$

where we used the fact $\frac{d}{d t} \tilde{q}(t)=\frac{d}{d t} \hat{q}(t)-\frac{d}{d t} q_{s s}=\hat{q}_{t}(t)$ and that $q_{s s}=$ $-A_{q}^{-1} c_{s s}$.

The difference in the (slowly) time varying case arises in the definition of the energy functional which is now given by

$$
\begin{aligned}
E(t)= & \gamma\left\{\sigma_{1}\left(q^{*} ; \epsilon(t), e(t)\right)+\left|e_{t}(t)\right|^{2}\right\}+2\left\langle\epsilon(t), e_{t}(t)\right\rangle \\
& +\sigma_{2}\left(q^{*} ; \epsilon(t), e(t)\right)+|\tilde{q}(t)|_{Q}^{2}+\beta|\bar{q}(t)|_{Q}^{2},
\end{aligned}
$$

for some $\beta>0$ to be defined later. The derivative $E_{t}(t)$ is given by

$$
\begin{aligned}
E_{t}(t)= & \gamma\left\{2 \sigma_{1}\left(q^{*} ; e(t), e_{t}(t)\right)+2\left\langle e_{t t}(t), e_{t}(t)\right\rangle\right\}+2\left\langle e_{t}(t), e_{t}(t)\right\rangle \\
& +2\left\langle e(t), e_{t t}(t)\right\rangle+2 \sigma_{2}\left(q^{*} ; e_{t}(t), e(t)\right)+2\left\langle\tilde{q}_{t}(t), \tilde{q}(t)\right\rangle_{Q} \\
& +2 \beta\left\langle\bar{q}_{t}(t), \bar{q}(t)\right\rangle_{Q} \\
= & -2 \gamma \sigma_{2}\left(q^{*} ; e_{t}(t), e_{t}(t)\right)-2 \sigma_{1}\left(q^{*} ; e(t), e(t)\right)+2\left|e_{t}(t)\right|^{2} \\
& +2 \sigma_{2}\left(\bar{q}(t) ; z_{t}(t), e(t)\right)+2 \sigma_{1}(\bar{q}(t) ; z(t), e(t)) \\
& +2 \gamma \sigma_{2}\left(\bar{q}(t) ; z_{t}(t), e_{t}(t)\right)+2 \gamma \sigma_{1}\left(\bar{q}(t) ; z(t), e_{t}(t)\right) \\
& -2 \gamma b\left(\bar{q}(t) ; f(t), e_{t}(t)\right)-2 b(\bar{q}(t) ; f(t), e(t))+2 \beta\left\langle A_{q} \bar{q}(t), \bar{q}(t)\right\rangle_{Q} \\
\leq & -2 \alpha_{0}\left(q^{*}\right)\|e(t)\|_{V_{1}}^{2}+\left\{-2 \gamma \beta_{0}\left(q^{*}\right)+2 K_{V_{2}}^{2}\right\}\left\|e_{t}(t)\right\|_{V_{2}}^{2} \\
& +2 \lambda|\bar{q}(t)|_{Q}\|e(t)\|_{V_{2}}+2 \gamma \lambda|\bar{q}(t)|_{Q}\left\|e_{t}(t)\right\|_{V_{2}}-\beta \kappa_{q}|\bar{q}(t)|_{Q}^{2} \\
\leq & -\left\{2 \alpha_{0}\left(q^{*}\right)-\lambda K_{V_{2}, V_{1}}^{2} \mu_{1}\right\}\|e(t)\|_{V_{1}}^{2} \\
& -\left\{2 \gamma \beta_{0}\left(q^{*}\right)-2 K_{V_{2}}^{2}-\gamma \lambda \mu_{2}\right\}\left\|e_{t}(t)\right\|_{V_{2}}^{2} \\
& -\left\{\beta \kappa_{q}-\left(\frac{1}{4 \mu_{1}}+\frac{\gamma}{4 \mu_{2}}\right) \lambda\right\}|\bar{q}(t)|_{Q}^{2} \\
\leq & -\rho_{0}\left\{\|e(t)\|_{V_{1}}^{2}+\left\|e_{t}(t)\right\|_{V_{2}}^{2}+|\bar{q}(t)|_{Q}^{2}\right\},
\end{aligned}
$$

where we used the identity $a \cdot b \leq a^{2} / 4 \mu_{1}+b^{2} \cdot \mu_{1}$. The equivalent of Lemma 3.1 is 
LEMMA 4.2. If $\beta, \mu_{1}, \mu_{2}$ are chosen so that the following are satisfied

$$
0<\mu_{1}<\frac{2 \alpha_{0}\left(q^{*}\right)}{\lambda K_{V_{2}}^{2}}, \quad 0<\mu_{2}<\frac{2 \beta_{0}\left(q^{*}\right)}{\lambda},
$$

and

$$
\beta>\frac{\lambda^{2}}{8 \kappa_{q}}\left[\frac{K_{V_{2}}^{2}}{\alpha_{0}\left(q^{*}\right)}+\frac{\gamma}{\beta_{0}\left(q^{*}\right)}\right],
$$

where $\gamma$ now satisfies

$$
\gamma>\max \left\{K_{V_{1}}, \frac{K_{V_{1}}}{\alpha_{0}\left(q^{*}\right)}, \frac{2 K_{V_{2}}^{2}}{2 \beta_{0}\left(q^{*}\right)-\lambda \mu_{2}}\right\},
$$

then there exist constants $\rho, \sigma>0$ such that for all $t>0$

$$
\begin{aligned}
\|e(t)\|_{V_{1}}^{2} & +\left|e_{t}(t)\right|^{2}+|\tilde{q}(t)|_{Q}^{2}+|\bar{q}(t)|_{Q}^{2} \\
& +\rho \int_{0}^{t}\left\{\|e(s)\|_{V_{1}}^{2}+\left\|e_{s}(s)\right\|_{V_{2}}^{2}+|\bar{q}(s)|_{Q}^{2}\right\} d s \leq \xi
\end{aligned}
$$

where $\xi=\sigma\left\{\|e(0)\|_{V_{1}}^{2}+\left|e_{t}(0)\right|^{2}+|\tilde{q}(0)|_{Q}^{2}+|\bar{q}(0)|_{Q}^{2}\right\}$.

The proof of Lemma 4.2 readily follows from equation (4.13) and using arguments similar to the ones in the proof of Lemma 3.1. We now establish the convergence of the state error.

THEOREM 4.3. Let $(q, z, f)$ be an admissible plant. If $\gamma$ satisfies the conditions in Lemma 4.2, then the energy functional E given by (4.12) is nonincreasing, and

$$
\lim _{t \rightarrow \infty}\|e(t)\|_{V_{1}}=0 \quad \text { and } \quad \lim _{t \rightarrow \infty}\left|e_{t}(t)\right|=0 .
$$

Proof. The proof of Theorem 4.3 is similar to the one given for the time invariant case with the exception of minor modifications and is therefore omitted.

Parameter convergence is again achieved by imposing the additional condition of persistence of excitation. If the plant $(q, z, f)$ is persistently excited (see Definition 3.3) then

$$
\lim _{t \rightarrow \infty}|\tilde{q}(t)|_{Q}=0
$$

The proof of parameter convergence is similar to the previous case of time invariant parameters and is summarized in Appendix $B$ of [3].

REMARK 4.4. The above theorem shows convergence of the parameter estimate $\hat{q}(t)$ to the steady state value $q_{s s}$ of the unknown parameter $q$. Since $|r(t)|_{Q}^{2}=\left|\left(\hat{q}(t)-q_{s s}\right)-\left(q(t)-q_{s s}\right)\right|_{Q}^{2}=|\tilde{q}(t)-\bar{q}(t)|_{Q}^{2} \leq 2|\tilde{q}(t)|_{Q}^{2}+2|\bar{q}(t)|_{Q}^{2}$, and since

$$
\lim _{t \rightarrow \infty}\left|q(t)-q_{s s}\right|_{Q}=0, \quad \text { by (4.10) and the assumption on } A_{q},
$$

then we have that

$$
\lim _{t \rightarrow \infty}|\hat{q}(t)-q(t)|_{Q}=0 .
$$


Further study is needed to see if the parameter estimate, $\hat{q}(t)$, can converge to the parameter $q(t)$ (in finite time) instead of its steady state value $q_{s s}$.

REMARK 4.5. Using equations (4.4), (4.10) and (4.13) it can be seen that it is not $A_{q}$ that is required to be known, but rather its coercivity bound $\kappa_{q}$ that is used in (4.13).

REMARK 4.6. A similar result can be established for the case of no damping and time varying parameters. Combination of results from the previous sections can give the conditions needed for the estimator of time varying systems with no damping.

\section{Finite Dimensional Approximation}

All the adaptive identification schemes presented in the previous sections are infinite dimensional and their application necessitates a finite dimensional approximation. We will outline a Galerkin approach in order to implement finite dimensional adaptive estimators and establish abstract convergence results. Even though the adaptive estimators require information on the full state, a rather unlikely scenario, the approximate estimators will be shown to adequately function with a finite dimensional information of the state of the system. This, in a way, can be viewed as an adaptive (finite dimensional) state and parameter estimator that uses a finite dimensional output of the system (this being a finite dimensional approximation of the system) to yield estimates of the state and the parameters of the actual infinite dimensional system.

For each $n=1,2, \ldots$, let $H^{n}$ be a finite dimensional subspace of $H$ with $H^{n} \subset V_{1}, n=1,2, \ldots$, and let $Q^{n}$ be a finite dimensional subspace of $Q$. We consider only the case of the non-symmetric bilinear form $\sigma_{2}$, the other two cases, the slowly time-varying systems and systems with no damping, being similar and therefore omitted. The Galerkin equations for $\hat{z}^{n}$ and $\hat{q}^{n}$ in $H^{n}$ and $Q^{n}$ corresponding to (2.9) and (2.10) are given by

$$
\begin{aligned}
& \left\langle\hat{z}_{t t}^{n}(t), \varphi^{n}\right\rangle+\Sigma_{2}\left(\hat{z}_{t}^{n}(t), \varphi^{n}\right)+\Sigma_{1}\left(\hat{z}^{n}(t), \varphi^{n}\right)+\sigma_{2}\left(\hat{q}^{n}(t) ; z_{t}(t), \varphi^{n}\right) \\
& +\sigma_{1}\left(\hat{q}^{n}(t) ; z(t), \varphi^{n}\right) \\
& =\quad b\left(\hat{q}^{n}(t) ; f(t), \varphi^{n}\right)+\Sigma_{2}\left(z_{t}(t), \varphi^{n}\right)+\Sigma_{1}\left(z(t), \varphi^{n}\right), \varphi^{n} \in H^{n}, t>0 \\
& \left\langle\hat{q}_{t}^{n}(t), p^{n}\right\rangle_{Q}+\sigma_{2}\left(p^{n} ; z_{t}(t), z(t)-\hat{z}^{n}(t)\right)+\sigma_{1}\left(p^{n} ; z(t), z(t)-\hat{z}^{n}(t)\right) \\
& -b\left(p^{n} ; f(t), z(t)-\hat{z}^{n}(t)\right)+\gamma \sigma_{2}\left(p^{n} ; z_{t}(t), z_{t}(t)-\hat{z}_{t}^{n}(t)\right) \\
& +\gamma \sigma_{1}\left(p^{n} ; z(t), z_{t}(t)-\hat{z}_{t}^{n}(t)\right)-\gamma b\left(p^{n} ; f(t), z_{t}(t)-\hat{z}_{t}^{n}(t)\right)=0 \\
& p^{n} \in Q^{n}, t>0, \\
& \hat{z}^{n}(0) \in H^{n}, \quad \hat{z}_{t}^{n}(0) \in H^{n}, \quad \hat{q}^{n}(0) \in Q^{n} .
\end{aligned}
$$

We make the following standard Galerkin approximation assumptions. First define the orthogonal projections $P^{n}: H \rightarrow H^{n}$ of $H$ onto $H^{n}$ and $P_{Q}^{n}: Q \rightarrow Q^{n}$ of $Q$ onto $Q^{n}$.

(H1) The finite dimensional subspaces $H^{n}$ satisfy $H^{n} \subset V_{1}$

(H2) The functions $\hat{\zeta}^{n}:=P^{n} \hat{z}$ and $\hat{\theta}^{n}:=P_{Q}^{n} \hat{q}$ with $\hat{\zeta}^{n} \in L_{2}\left(0, T ; H^{n}\right)$ and $\hat{\theta}^{n}:=P_{Q}^{n} \hat{q} \in L_{2}\left(0, T ; Q^{n}\right)$ are such that 
(i) $\hat{\zeta}^{n} \rightarrow \hat{z}$ in $C\left(0, T ; V_{1}\right)$,

(ii) $\frac{d}{d t} \hat{\zeta}^{n} \rightarrow \frac{d}{d t} \hat{z}$ in $C(0, T ; H)$ and $L_{2}\left(0, T ; V_{2}\right)$,

(iii) $\hat{\theta}^{n} \rightarrow \hat{q}$ in $C(0, T ; Q)$.

Using the above assumptions we prove the following convergence result. Theorem 5.1. Assume that assumptions $(H 1)$ - $(H 2)$ hold, and $(q, z, f)$ satisfies Definition 2.1. Let $(\hat{q}, \hat{z})$ be the solution to the initial value problem (2.9) - (2.11), and for each $n=1,2, \ldots$, let $\left(\hat{q}^{n}, \hat{z}^{n}\right)$ be the solution to the initial value problem (5.1) - (5.3) with

$$
\begin{gathered}
\hat{z}^{n}(0)=\hat{\zeta}^{n}(0)=P^{n} \hat{z}(0), \quad \frac{d}{d t} \hat{z}^{n}(0)=\frac{d}{d t} \hat{\zeta}^{n}(0)=P^{n} \frac{d}{d t} \hat{z}(0), \\
\hat{q}^{n}(0)=\hat{\theta}^{n}(0)=P_{Q}^{n} \hat{q}(0) .
\end{gathered}
$$

Then

$$
\begin{aligned}
& \text { (i) } \hat{z}^{n} \rightarrow \hat{z} \quad \text { in } \quad C\left(0, T ; V_{1}\right) \\
& \text { (ii) } \frac{d}{d t} \hat{z}^{n} \rightarrow \frac{d}{d t} \hat{z} \quad \text { in } \quad C(0, T ; H) \text { and } L_{2}\left(0, T ; V_{2}\right), \text { and } \\
& \text { (iii) } \hat{q}^{n} \rightarrow \hat{q} \quad \text { in } \quad C(0, T ; Q) .
\end{aligned}
$$

Proof. Since the proof is rather lengthy and technical, it is summarized in Appendix $\mathrm{C}$ of [3]. Even though the scheme here is similar to the one presented in [15], Theorem 5.1 does not impose any assumptions on $\frac{d^{2}}{d t^{2}} \hat{\zeta}^{n}$, and $\frac{d}{d t} \hat{\theta}^{n}$. The proof can be given under relaxed conditions by following ideas in [11] (see also Chapter 5 of [10]).

The above finite dimensional estimator requires the state of the system, $z$, which lies in the infinite dimensional space $V_{1}$. Therefore, it will be more convenient to replace it in $(5.1)$ - $(5.3)$ by a finite dimensional approximation, $z^{n}$. In order to present an approximation result that would use a finite dimensional approximation of the plant, we need to impose some conditions on the plant, as was similarly done for the symmetric case in [15].

(H3) There exists a constant $s_{1}>0$ such that

$$
\left|\sigma_{1}(p ; \varphi, \psi)\right| \leq s_{1}|p|_{Q}\|\varphi\|_{V_{1}}\|\psi\|_{V_{1}}, \quad p \in Q, \quad \varphi, \psi \in V_{1} .
$$

(H4) There exists a constant $s_{2}>0$ such that

$$
\left|\sigma_{2}(p ; \varphi, \psi)\right| \leq s_{2}|p|_{Q}\|\varphi\|_{V_{2}}\|\psi\|_{V_{2}}, \quad p \in Q, \quad \varphi, \psi \in V_{2} .
$$

(H5) There exists a constant $b_{1}>0$ such that

$$
|b(p ; \varphi, \psi)| \leq b_{1}|p|_{Q}|\varphi|\|\psi\|_{V_{1}}, \quad p \in Q, \quad \varphi \in H, \quad \psi \in V_{1} .
$$

(H6) For the plant $(q, z, f)$ there exists $z^{n} \in C^{1}\left(0, T ; H^{n}\right)$ such that

(i) $z^{n} \rightarrow z$ in $C\left(0, T ; V_{1}\right)$,

(ii) $\dot{z}^{n} \rightarrow \dot{z}$ in $C\left(0, T ; V_{1}\right)$.

Theorem 5.2. Assume that $(q, z, f)$ is an admissible plant and that $(H 1)$ (H6) are satisfied. Let $(\hat{q}, \hat{z})$ be the solution to (2.9) - (2.11), and for each $n=1,2, \ldots$, let $\left(\hat{q}^{n}, \hat{z}^{n}\right)$ be the solution to (5.1) - (5.3) with z replaced by ESAIM: COCV, MAY 1998, Vol. 3, 133-162 
$z^{n}$ and $\dot{z}$ by $\dot{z}^{n}$. Then

(i) $\hat{z}^{n} \rightarrow \hat{z}$ in $C\left(0, T ; V_{1}\right)$,

(ii) $\frac{d}{d t} \hat{z}^{n} \rightarrow \frac{d}{d t} \hat{z} \quad$ in $\quad C(0, T ; H)$ and $L_{2}\left(0, T ; V_{2}\right)$, and

(iii) $\hat{q}^{n} \rightarrow \hat{q} \quad$ in $C(0, T ; Q)$.

Proof. The proof is also summarized in Appendix C of [3].

REMARK 5.3. As it was observed in [15], if we choose $P_{V_{2}}^{n}: V_{2} \rightarrow H^{n}$ be the orthogonal projection of $V_{2}$ onto $H^{n}$ with respect to the standard $V_{2}$ inner product and set

$$
z^{n}:=P_{V_{2}}^{n} z
$$

then we see that

$$
\begin{array}{lll}
\sigma_{1}\left(p ; P_{V_{2}}^{n} z, \varphi\right) \equiv \sigma_{1}(p ; z, \varphi) & \text { for } & \varphi \in H^{n} \\
\sigma_{2}\left(p ; P_{V_{2}}^{n} \dot{z}, \varphi\right) \equiv \sigma_{2}(p ; \dot{z}, \varphi) & \text { for } & \varphi \in H^{n}, \\
\Sigma_{1}\left(P_{V_{2}}^{n} z, \varphi\right) \equiv \Sigma_{1}(z, \varphi) & \text { for } & \varphi \in H^{n}, \quad \text { and } \\
\Sigma_{2}\left(P_{V_{2}}^{n} \dot{z}, \varphi\right) \equiv \Sigma_{2}(\dot{z}, \varphi) & \text { for } & \varphi \in H^{n} .
\end{array}
$$

Indeed, the above make sense since $z \in L_{2}\left(0, T ; V_{1}\right)$ (and $z \in L_{2}\left(0, T ; V_{2}\right)$ ), and $\dot{z} \in L_{2}\left(0, T ; V_{2}\right)$ were assumed as part of the existence of a weak solution to the plant $(2.2),(2.3)$.

REMARK 5.4. From the conclusion of Theorem 5.2 and Remark 5.3 above, we can see that if only a finite dimensional approximation $\left(z^{n}, \dot{z}^{n}\right)$ of the plant $(z, \dot{z})$ is available and used in $(5.1)$ - (5.3), then this may be viewed as an adaptive state and parameter estimator that uses a part of the state $(z, \dot{z})$ with the output $y(t) \in H^{n} \times H^{n}$ of the system having the specific form

$$
y(t)=\mathcal{C}\left(\begin{array}{c}
z(t) \\
\dot{z}(t)
\end{array}\right)=\left(\begin{array}{c}
P_{V_{2}}^{n} z(t) \\
P_{V_{2}}^{n} \dot{z}(t)
\end{array}\right)=\left(\begin{array}{c}
z^{n}(t) \\
\dot{z}^{n}(t)
\end{array}\right) \in H^{n} \times H^{n},
$$

where the output (or observation) operator $\mathcal{C}: V_{2} \times V_{2} \rightarrow H^{n} \times H^{n}$.

\section{Examples and Numerical Simulations}

As an example of a system with non-symmetric damping, we study the 2-D structural acoustic model with piezoceramic actuators presented in [4] which indeed motivated the efforts of this paper. This 2-D structural acoustic model investigated here represents a "linearized slice" of a full 3-D acoustic cavity/plate structure currently being used in experiments in the Acoustics Division at NASA Langley Research Center, see [10] for further details.

The equations of motion for the 2-D linearized structural acoustic model are given by

$$
\begin{aligned}
& \phi_{t t}=c^{2} \Delta \phi+d \Delta \phi_{t}, \quad(x, y) \in \Omega, t>0 \\
& \nabla \phi \cdot \hat{n}=0, \quad(x, y) \in \Gamma, t>0, \\
& \nabla \phi(t, x, 0) \cdot \hat{n}=w_{t}(t, x), \quad 0<x<a, t>0, \\
& \text { ESAIM: Cocv, MAY 1998, Vol. } 3,133-162
\end{aligned}
$$




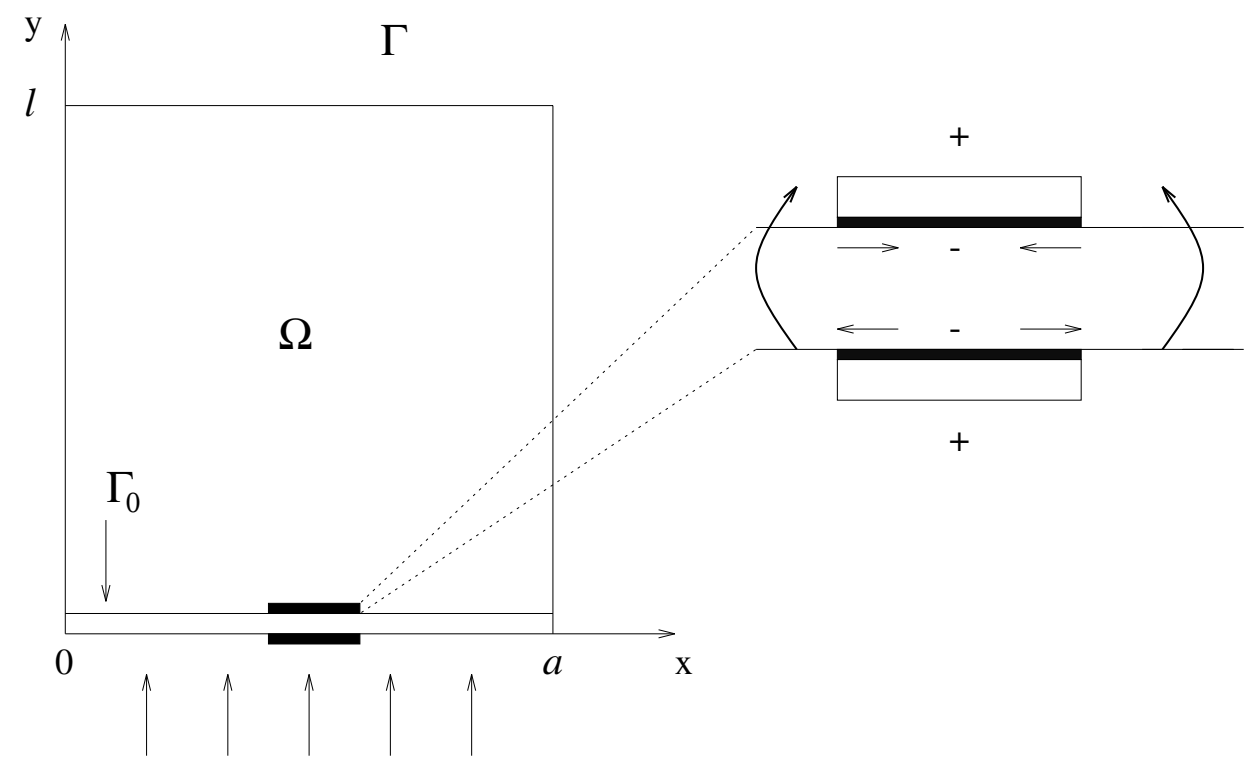

(a)

(b)

FiguRE 1. (a) Acoustic cavity with piezoceramic patches,

(b) Piezoceramic patch excitation.

$$
\begin{gathered}
\rho_{b} w_{t t}+\frac{\partial^{2}}{\partial x^{2}}\left(E I \frac{\partial^{2} w}{\partial x^{2}}+c_{D} I \frac{\partial^{3} w}{\partial x^{2} \partial t}\right) \\
=-\rho_{f} \phi_{t}(t, x, 0)+f(t, x)+\frac{\partial^{2}}{\partial x^{2}}\left(\mathcal{K}^{B} \chi_{\left[x_{1}, x_{2}\right]} u(t)\right), \quad 0<x<a, t>0 \\
w(t, 0)=\frac{\partial w}{\partial x}(t, 0)=w(t, a)=\frac{\partial w}{\partial x}(t, a)=0, \quad t>0 \\
\phi(0, x, y)=\phi_{0}(x, y), \quad w(0, x)=w_{0}(x), \\
\phi_{t}(0, x, y)=\phi_{1}(x, y), \quad w_{t}(0, x)=w_{1}(x)
\end{gathered}
$$

where $\phi(t, x, y)$ denotes the cavity potential, $\rho_{f} \phi_{t}(t, x, y)$ the cavity pressure with $\rho_{f}$ being the equilibrium fluid density. The fluid constants $c$ and $d$ denote the speed of sound in the fluid and damping coefficient, respectively. The patch parameters $u(t), \chi_{\left[x_{1} x_{2}\right]}(x)$ and $\mathcal{K}^{B}$ denote the voltage applied to the patch, the characteristic function for the location of the patch and patch parameter, see $[5,9]$.

The beam transverse displacement is denoted by $w(t, x)$ and the beam parameters $\rho_{b}, E I$ and $c_{D} I$ represent the linear mass density, stiffness and damping, respectively. In this note the piezoceramic material parameter $\mathcal{K}^{B}$, as well as the beam parameters $E I$ and $c_{D} I$ are considered to be unknown and must be estimated adaptively (on-line).

The domain is given by $\Omega=[0, a] \times[0, l]$ and the boundary is denoted by $\Gamma$ (hard walls) and $\Gamma_{0}$ (perturbable boundary-beam), see Figure 1.

In order to pose the problem in a variational form which is required for our approach to adaptive parameter estimation and approximation, (see [10]), the state is taken to be $z=(\phi, w)$ in the state space $H=\bar{L}_{2}(\Omega) \times L_{2}\left(\Gamma_{0}\right)$ ESAIM: COCV, MAY 1998, VOL. 3, 133-162 
with the energy inner product

$$
\left\langle\left(\begin{array}{c}
\phi \\
w
\end{array}\right),\left(\begin{array}{c}
\xi \\
\eta
\end{array}\right)\right\rangle_{H}=\int_{\Omega} \rho_{f} \phi \xi d \omega+\int_{\Gamma_{0}} \rho_{b} w \eta d \gamma
$$

Here, $\bar{L}_{2}(\Omega)$ is the quotient space of $L_{2}$ over the constant functions. The quotient space is used because the potentials are determined only up to a constant, again see [10]. We also define the Hilbert space $V_{1}=\overline{H^{1}}(\Omega) \times H_{0}^{2}\left(\Gamma_{0}\right)$ where $\overline{H^{1}}(\Omega)$ is the quotient space of $H^{1}(\Omega)$ over the constant functions and $H_{0}^{2}(\Gamma)=\left\{\psi \in H^{2}(\Gamma): \psi(x)=\psi^{\prime}(x)=0\right.$ at $\left.x=0, a\right\}$. The $V_{1}$ inner product is taken as

$$
\left\langle\left(\begin{array}{c}
\phi \\
w
\end{array}\right),\left(\begin{array}{c}
\xi \\
\eta
\end{array}\right)\right\rangle_{V_{1}}=\int_{\Omega} \nabla \phi \cdot \nabla \xi d \omega+\int_{\Gamma_{0}} D^{2} w D^{2} \eta d \gamma
$$

In this case the parameter $q$ is taken to be $q=\left\{E I(\cdot), c_{D} I(\cdot), \mathcal{K}^{B}\right\}$. For $q \in Q$, we define bilinear forms $\sigma_{i}(q ; \cdot, \cdot): V_{1} \times V_{1} \rightarrow \mathbb{R}^{1}, i=1,2$, on $\mathrm{V}$ by

$$
\begin{gathered}
\sigma_{1}(q ; \Phi, \Psi)=\int_{\Omega} \rho_{f} c^{2} \nabla \phi \cdot \nabla \xi d \omega+\int_{\Gamma_{0}} E I D^{2} w D^{2} \eta d \gamma, \\
\sigma_{2}(q ; \Phi, \Psi)=\int_{\Omega} \rho_{f} d \nabla \phi \cdot \nabla \xi d \omega+\int_{\Gamma_{0}}\left\{c_{D} I D^{2} w D^{2} \eta+\rho_{f}(\phi \eta-w \xi)\right\} d \gamma,
\end{gathered}
$$

where $\Phi=(\phi, w)$ and $\Psi=(\xi, \eta)$ are in $V_{1}$. In addition, for each $q \in Q$, we define the bilinear form $b(q ; \cdot, \cdot): H \times V_{1} \rightarrow \mathbb{R}^{1}$ by

$$
b(q ; \Theta, \Psi)=\int_{\Gamma_{0}} \mathcal{K}^{B} \nu D^{2} \eta d \gamma, \quad \Theta=(\mu, \nu) \in H, \Psi \in V_{1} .
$$

In the work reported here, we followed the approach in $[5],[8],[9]$, using a Galerkin approximation scheme to approximate the beam/cavity system. This was achieved by discretizing the potential and beam displacement in terms of spectral and spline expansions, respectively.

Our scheme for the second order formulation approximating (2.2) was of dimension $N$ where $N=m+n-1$ with $n-1$ modified cubic splines used for the beam approximation and $m$ tensor products of Legendre polynomials used for the cavity approximation (see [5] for complete details). Using a standard Galerkin scheme, we choose a sequence of finite dimensional subspaces $H^{N} \subset V_{1}$ with projections $\mathcal{P}^{N}: H^{N} \rightarrow H$.

To illustrate the approximation technique mentioned above let $\left\{B_{i}^{n}\right\}_{i=1}^{n-1}$ denote the 1-D basis functions which are used to discretize the beam and let $\left\{B_{i}^{m}\right\}_{i=1}^{m}, m=\left(m_{x}+1\right) \cdot\left(m_{y}+1\right)-1$, denote the 2 -D basis functions which are used in the cavity. The $n-1$ and $m$ dimensional approximating subspaces are then taken to be $H_{b}^{n}=\operatorname{span}\left\{B_{i}^{n}\right\}_{i=1}^{n-1}$ and $H_{c}^{m}=\left\{B_{i}^{m}\right\}_{i=1}^{m}$, respectively. Using the definition of $N$ above, the approximating state space is $H^{N}=H_{c}^{m} \times H_{b}^{n}$. The restriction of the infinite dimensional system to the space $H^{N}$ yields for $\Psi=(\xi, \lambda)$ the following

$$
\left\langle z_{t t}^{N}(t), \Psi\right\rangle_{X}+\sigma_{2}\left(q ; z_{t}^{N}(t), \Psi\right)+\sigma_{1}\left(q ; z^{N}(t), \Psi\right)=\int_{\Gamma_{0}} \mathcal{K}^{B} \chi_{\left[x_{1}, x_{2}\right]}(x) u(t) D^{2} \lambda d \gamma
$$


When $\Psi$ is chosen in $H^{N}$ and the approximate beam and cavity solutions are taken to be

$$
w^{N}(t, x)=\sum_{i=1}^{n-1} w_{i}^{N}(t) B_{i}^{n}(x) \quad \text { and } \quad \phi^{N}(t, x, y)=\sum_{i=1}^{m} \phi_{i}^{N}(t) B_{i}^{m}(x, y)
$$

respectively, this yields the system

$$
\begin{gathered}
M_{2}^{N} z_{t t}^{N}(t)+A_{2}^{N}(q) z_{t}^{N}(t)+A_{1}^{N}(q) z^{N}(t)=B^{N}(q) u(t)+F^{N}(t), \\
M_{1}^{N} z^{N}(0)=g_{1}^{N}, \quad M_{2}^{N} z_{t}^{N}(0)=g_{2}^{N} .
\end{gathered}
$$

Here

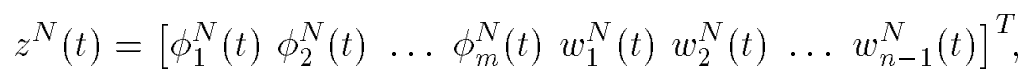

with $z_{t}^{N}(t)$ defined analogously, denote the $N \times 1=(m+n-1) \times 1$ approximate state vector coefficients. The structure of the approximate system's matrices are given in detail in [3] (see also [5]).

For our computational tests, we chose physical parameters based on our experimental efforts. The parameter choices $a=0.6 \mathrm{~m}, l=1 \mathrm{~m}, \rho_{f}=$ $1.21 \mathrm{~kg} / \mathrm{m}^{3}, c^{2}=117649 \mathrm{~m}^{2} / \mathrm{sec}^{2}, \rho_{b}=1.35 \mathrm{~kg} / \mathrm{m}, x_{1}=0.20$ and $x_{2}=0.40$ are reasonable for a $.6 \mathrm{~m}$ by $1 \mathrm{~m}$ cavity in which the bounding end beam has a centered piezoceramic patch covering $1 / 3$ of the beam. The beam is assumed to have width and thickness $.1 \mathrm{~m}$ and $0.005 \mathrm{~m}$, respectively.

For a simple set of runs, we used 4 cavity basis functions $\left(m_{x}=m_{y}=4\right)$ and 7 beam basis functions. Therefore $m=24$ and $n=8$ thus giving $N=$ 31. The dimension of the overall system (both plant and state estimator) is $4 N$.

It is worth mentioning that due to the structure of the bilinear form $\sigma_{2}$, the adaptive estimator utilized in the numerical studies below, was constructed via the method described by $\left(S_{1}\right)$ in Remark 2.2 with $\rho_{f}^{*} \equiv 0$ and a nonzero $c_{D} I^{*}$.

\subsection{Constant parameters}

As a first test of our scheme, we attempt to identify all three parameters, $\left\{E I(\cdot), c_{D} I(\cdot), \mathcal{K}^{B}(\cdot)\right\}$, which are assumed to be constant in time and space. In this case the actual parameters to be identified adaptively are

$$
E I=73.96 \mathrm{Nm}^{2}, \quad c_{D} I=0.001 \mathrm{kgm}^{3} / \mathrm{sec}, \quad \mathcal{K}^{B}=0.0067 \mathrm{~N} / \mathrm{m}^{2} V,
$$

and the tuning parameters

$$
E I^{*}(x)=80 \mathrm{Nm}^{2}, \quad c_{D} I^{*}(x)=0.1 \mathrm{kgm}^{3} / \mathrm{sec}, \quad 0 \leq x \leq 0.6 \mathrm{~m}, \quad \gamma=500 .
$$

Due to the structure of the damping form $\sigma_{2}$, we used the scheme $\left(S_{1}\right)$ in Remark 2.2 with $\rho_{f}^{*}=0$ in the selection of the tuning parameters.

The $Q$-inner product is taken to be the weighted Euclidean product on $\mathbb{R}^{3}$ given by

$$
\langle q, p\rangle_{Q}=\frac{1}{\alpha_{1}} q_{1} \cdot p_{1}+\frac{1}{\alpha_{2}} q_{2} \cdot p_{2}+\frac{1}{\alpha_{3}} q_{3} \cdot p_{3},
$$

$q=\left(q_{1}, q_{2}, q_{3}\right), p=\left(p_{1}, p_{2}, p_{3}\right) \in Q$, where the weights $\alpha_{i}, i=1,2,3$ are given by

$$
\alpha_{1}=2000, \quad \alpha_{2}=0.02, \quad \alpha_{3}=5 .
$$


The initial conditions for the plant state and the state estimator are taken to be zero and the initial guesses for the three parameters are

$$
\widehat{E I}(0)=55 \mathrm{Nm}^{2}, \quad \widehat{c_{D} I}(0)=0.0005 \mathrm{kgm}^{3} / \mathrm{sec}, \quad \widehat{\mathcal{K}^{B}}(0)=0.0090 \mathrm{~N} / \mathrm{m}^{2} V .
$$

In Figure 2(a)-(c), we see both the actual (dashed) and the estimated (solid) values of the three parameters. All three parameters converge after three seconds to within $2 \%$ of the actual value. The overshoot appearing in the estimate of $\widehat{c_{D} I}(t)$ can be reduced by tuning the parameters $E I^{*}(x), c_{D} I^{*}(x)$ and the gains $\gamma$ and $\alpha_{i}, i=1,2,3$. The high frequency oscillations observed in the first two seconds of the time history of $\widehat{\mathcal{K}^{B}}(t)$ was also observed in a similar study by Rosen and Demetriou in [24] for the adaptive identification of a flexible cantilevered Euler-Bernoulli beam.

\subsection{Functional parameters}

Continuing with our test, we now assume that $E I(\cdot)$ and $c_{D} I(\cdot)$ are spatially dependent and that $\mathcal{K}^{B}$ is a constant. The actual values are taken to be

$$
\begin{gathered}
E I(x)= \begin{cases}73.96 \mathrm{Nm}^{2} & \text { if } x \in[0.0,0.2) \\
125.4 \mathrm{Nm}^{2} & \text { if } x \in[0.2,0.4), \\
73.96 \mathrm{Nm}^{2} & \text { if } x \in(0.4,0.6]\end{cases} \\
c_{D} I(x)= \begin{cases}0.00100 \mathrm{kgm}^{3} / \mathrm{sec} & \text { if } x \in[0.0,0.2) \\
0.00125 \mathrm{kgm}^{3} / \mathrm{sec} & \text { if } x \in[0.2,0.4), \\
0.00100 \mathrm{kgm}^{3} / \mathrm{sec} & \text { if } x \in(0.4,0.6]\end{cases}
\end{gathered}
$$

and $\mathcal{K}^{B}=0.0067 \mathrm{~N} / \mathrm{m}^{2} V$. The tuning parameters are

$$
\begin{gathered}
E I^{*}(x)= \begin{cases}80.00 \mathrm{Nm}^{2} & \text { if } x \in[0.0,0.2) \\
135.0 \mathrm{Nm}^{2} & \text { if } x \in[0.2,0.4), \\
80.00 \mathrm{Nm}^{2} & \text { if } x \in(0.4,0.6]\end{cases} \\
c_{D} I^{*}(x)= \begin{cases}0.01 \mathrm{kgm}^{3} / \mathrm{sec} & \text { if } x \in[0.0,0.2) \\
0.01 \mathrm{kgm}^{3} / \mathrm{sec} & \text { if } x \in[0.2,0.4), \\
0.01 \mathrm{kgm}^{3} / \mathrm{sec} & \text { if } x \in(0.4,0.6]\end{cases}
\end{gathered}
$$

and $\gamma=500$, while the initial estimates are

$$
\begin{aligned}
& \widehat{E I}(x, 0)= \begin{cases}66.00 \mathrm{Nm}^{2} & \text { if } x \in[0.0,0.2) \\
110.0 \mathrm{Nm}^{2} & \text { if } x \in[0.2,0.4), \\
66.00 \mathrm{Nm}^{2} & \text { if } x \in(0.4,0.6]\end{cases} \\
& \widehat{c_{D} I}(x, 0)= \begin{cases}0.0009 \mathrm{kgm}^{3} / \mathrm{sec} & \text { if } x \in[0.0,0.2) \\
0.0011 \mathrm{kgm}^{3} / \mathrm{sec} & \text { if } x \in[0.2,0.4), \\
0.0009 \mathrm{kgm}^{3} / \mathrm{sec} & \text { if } x \in(0.4,0.6]\end{cases} \\
& \text { ESAIM: CoCv, MAY 1998, Vol. 3, 133-162 }
\end{aligned}
$$


and $\widehat{\mathcal{K}^{B}}(0)=0.0075 \mathrm{~N} / \mathrm{m}^{2} V$. For this case, the $Q$ - inner product is taken to be

$$
\begin{aligned}
\langle q(x), p(x)\rangle_{Q}= & \int_{0}^{0.6}\left(\frac{q_{11} p_{11}}{\alpha_{11}} \chi_{[0,0.2]}(x)+\frac{q_{12} p_{12}}{\alpha_{12}} \chi_{[0.2,0.4]}(x)\right. \\
& +\frac{q_{13} p_{13}}{\alpha_{13}} \chi_{[0.4,0.6]}(x)+\frac{q_{21} p_{21}}{\alpha_{21}} \chi_{[0,0.2]}(x) \\
& \left.+\frac{q_{22} p_{22}}{\alpha_{22}} \chi_{[0.2,0.4]}(x)+\frac{q_{23} p_{23}}{\alpha_{23}} \chi_{[0.4,0.6]}(x)\right) d x+\frac{q_{3} p_{3}}{\alpha_{3}},
\end{aligned}
$$

with the weights given by

$$
\alpha_{11}=\alpha_{12}=\alpha_{13}=3500, \quad \alpha_{21}=\alpha_{22}=\alpha_{23}=0.2, \quad \alpha_{3}=0.5 .
$$

Figure 3(a)-(c) shows the actual (solid) value for $E I(\cdot), c_{D} I(\cdot)$, and $\mathcal{K}^{B}$, the time history for $\widehat{\mathcal{K}^{B}}(t)$ and the time estimates at 0 (dashed) and at $2.5 \mathrm{sec}$ (dotted) for $\widehat{E I}(x, t)$ and $\widehat{c_{D} I}(x, t)$. The functional parameters converge to the true values after about 2.5 seconds within a $2 \%$ of the actual values. It should be noted that the stiffness functional parameter $\widehat{E I}(x, t)$ (Figure $3(\mathrm{a})$ ) converges to the actual parameter $E I(x)$ to within a $0.1 \%$ in less that 2.5 seconds which explains why the two graphs are indistinguishable. Finally, the piezoceramic parameter $\widehat{\mathcal{K}^{B}}(t)$ converges within a $5 \%$ of the actual value in 3 sec. Once again, the high frequency oscillations observed above, are also present in the time estimate of $\mathcal{K}^{B}$.

\subsection{Slowly time varying parameters}

In this example, we attempt to identify the two time varying parameters

$$
\frac{d}{d t} E I(t)=-\frac{1}{4} E I(t)+20, \quad \frac{d}{d t} c_{D} I(t)=-\frac{1}{4} c_{D} I(t)+0.000275
$$

where $E I_{s s}=80 \mathrm{Nm}^{2}, E I(0)=73.96 \mathrm{Nm}^{2}, \widehat{E I}(0)=68.00 \mathrm{Nm}^{2}$, and $c_{D} I_{s s}=11.0 \times 10^{-4} \mathrm{kgm}^{3} / \mathrm{sec}, c_{D} I(0)=10.0 \times 10^{-4} \mathrm{kgm}^{3} / \mathrm{sec}, \widehat{c_{D} I}(0)=$ $11.5 \times 10^{-4} \mathrm{kgm}^{3} / \mathrm{sec}$. The parameter $\mathcal{K}^{B}=0.0067 \mathrm{~N} / \mathrm{m}^{2} \mathrm{~V}$ is assumed to be known. In this case the tuning parameters and adaptive gains are

$$
E I^{*}=80 \mathrm{Nm}^{2}, \quad c_{D} I^{*}=0.01 \mathrm{kgm}^{3} / \mathrm{sec}, \quad \gamma=\alpha_{1}=1000, \quad \alpha_{2}=0.0003,
$$

Figure 4 shows the time estimates of $E I(t)$ and $c_{D} I(t)$. The time estimate $\widehat{E I}(t)$ of the time varying stiffness parameter converges to the actual value within $1 \mathrm{sec}$ whereas the time estimate $\widehat{c_{D} I}(t)$ of the damping parameter converges much later at around 5 sec to within a $2 \%$ of the actual time varying $c_{D} I(t)$.

\subsection{Systems With no DamPing}

The absence of damping allowed the use of the adaptive scheme (2.19), (2.20) with $\sigma_{2}=\sigma_{1}$. For this case the parameter $c_{D} I(x) \equiv 0$ for $0 \leq x \leq L$, and $\mathcal{K}^{B}=0.0067 \mathrm{~N} / \mathrm{m}^{2} V$ is assumed to be known. The parameter to be estimated is $E I=73.96 \mathrm{Nm}^{2}$ and the initial guess is $\widehat{E I}(0)=68 \mathrm{Nm}^{2}$. The tuning parameters and gains (using the scheme given by $(2.19),(2.20)$ ) are

$$
E I^{*}=80 \mathrm{Nm}^{2}, \quad c_{D} I^{*}=0.01 \mathrm{kgm}^{3} / \mathrm{sec}, \quad \gamma=\alpha_{1}=1000 .
$$




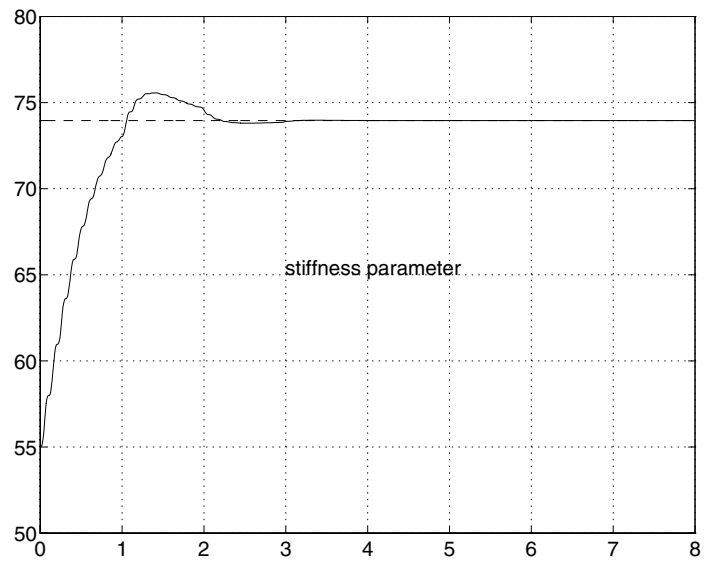

(a)

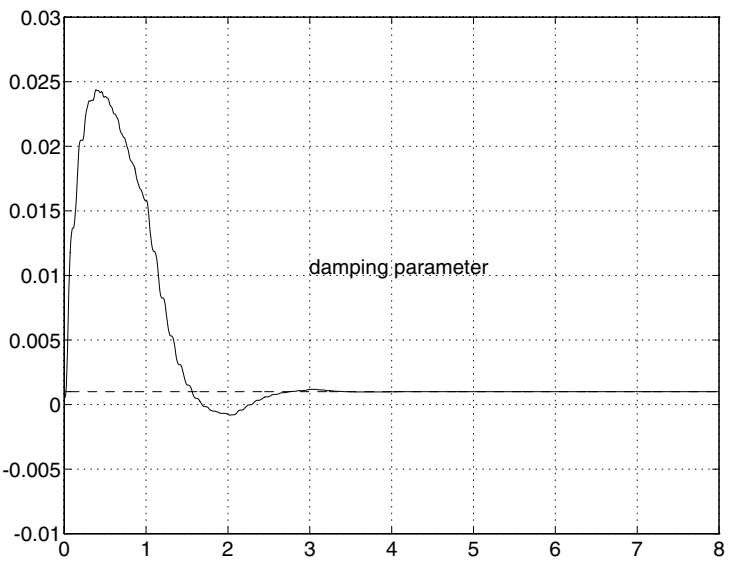

(b)

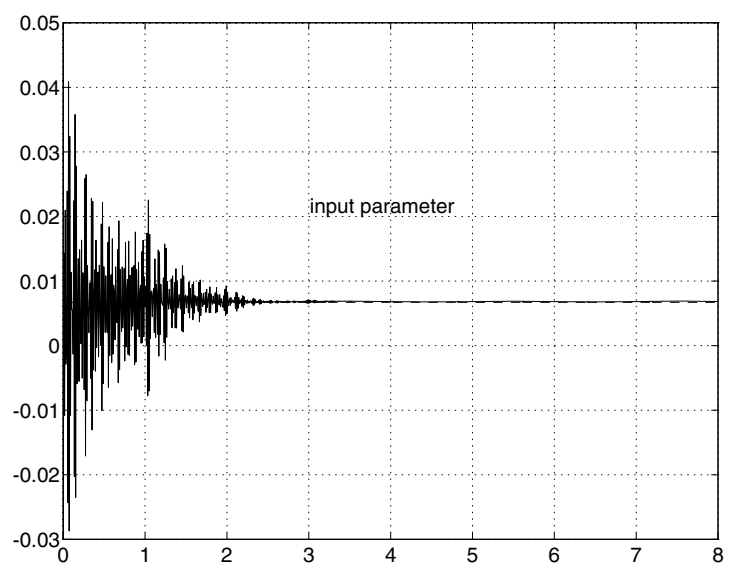

(c)

Figure 2. Actual and estimated parameters; constant (a)(c) (example 6.1). 


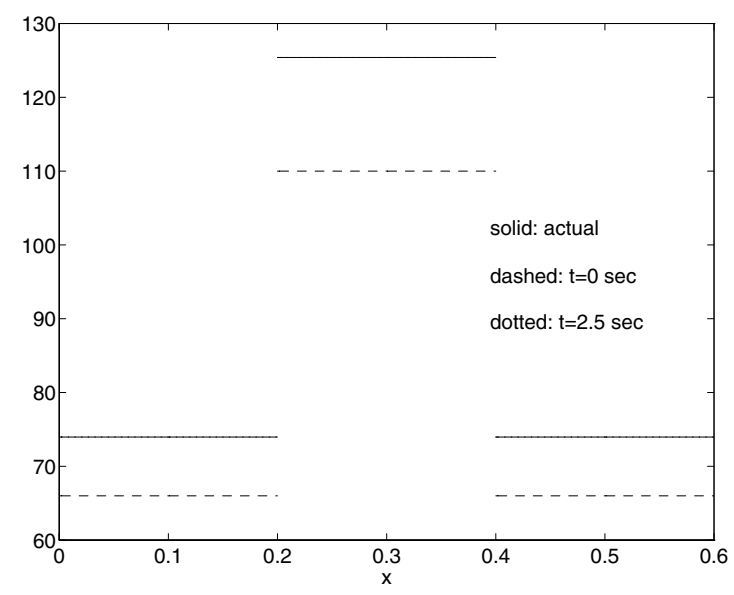

(a)

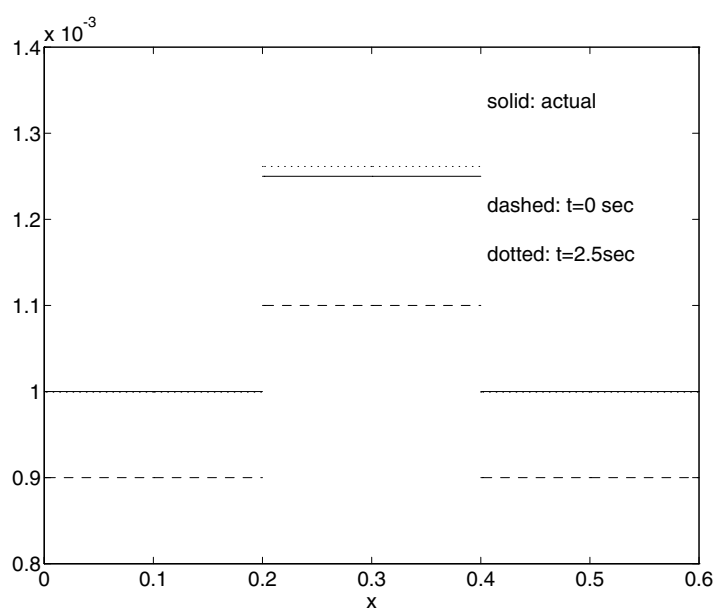

(b)

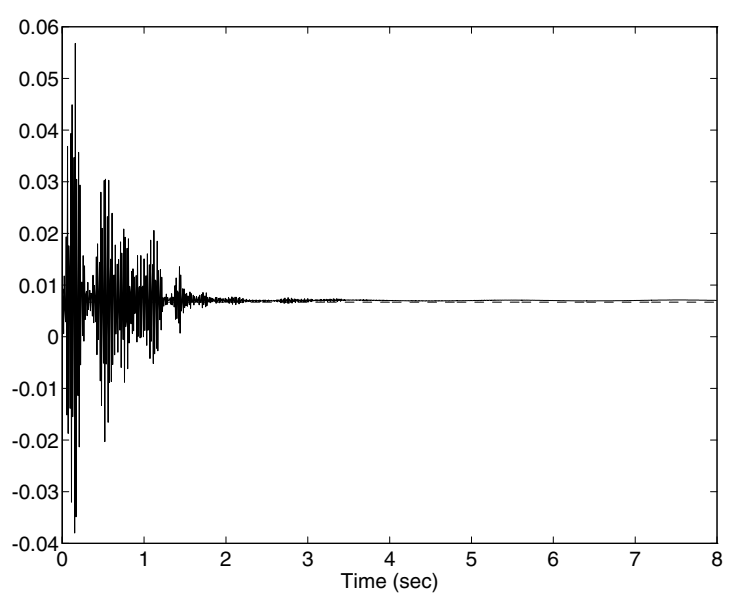

(c)

Figure 3. Actual and estimated parameters; functional (a)(c) (example 6.2). 


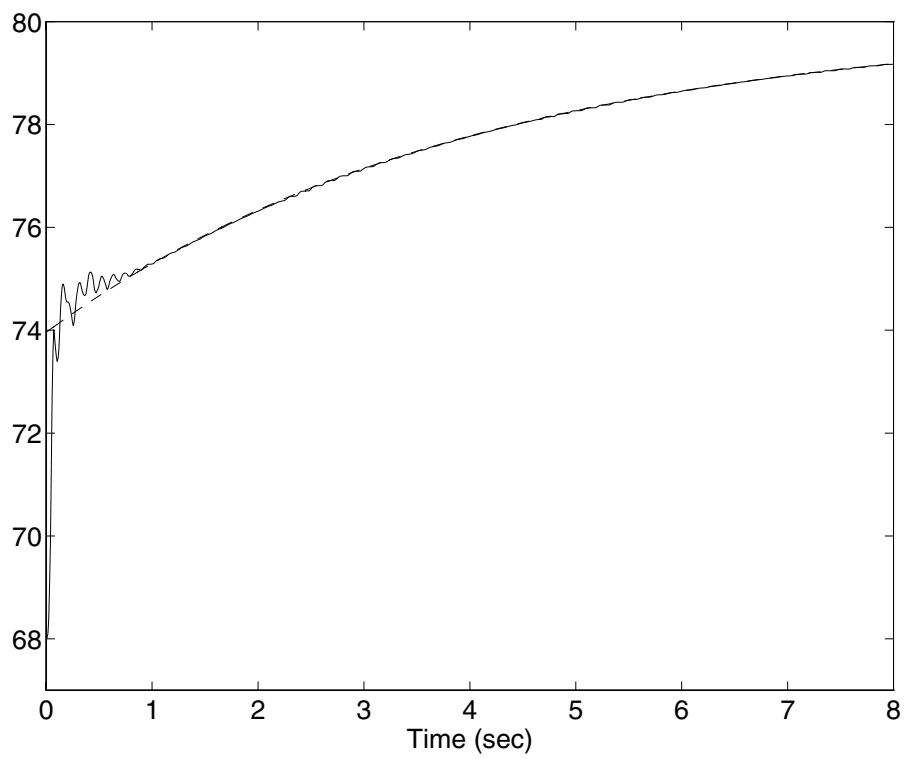

(a)

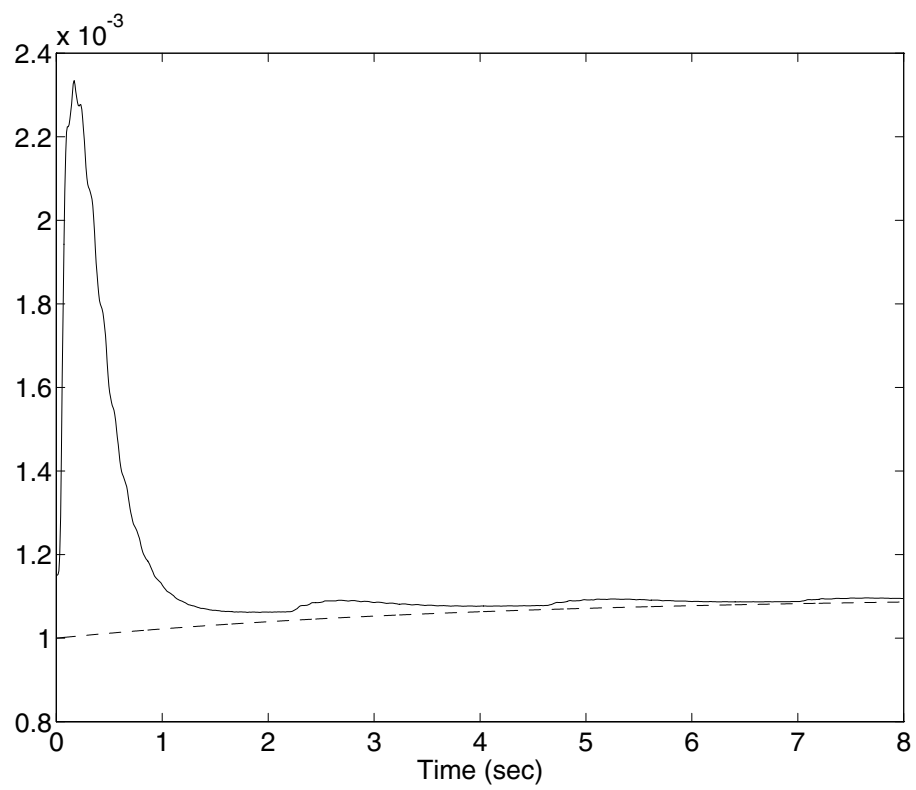

(b)

FiguRE 4. Actual and estimated values of time varying parameters; (a) stiffness $E I(t)$ (dashed) and $\widehat{E I}(t)$ (solid), (b) damping $c_{D} I(t)$ (dashed) and $\widehat{c_{D} I}(t)$ (solid).

The time estimate of $E I$ converges to a $0.5 \%$ of the actual value in $1 / 2$ sec as seen in Figure 5. Even when the system has no damping at all, the estimator can still estimate the parameter. 


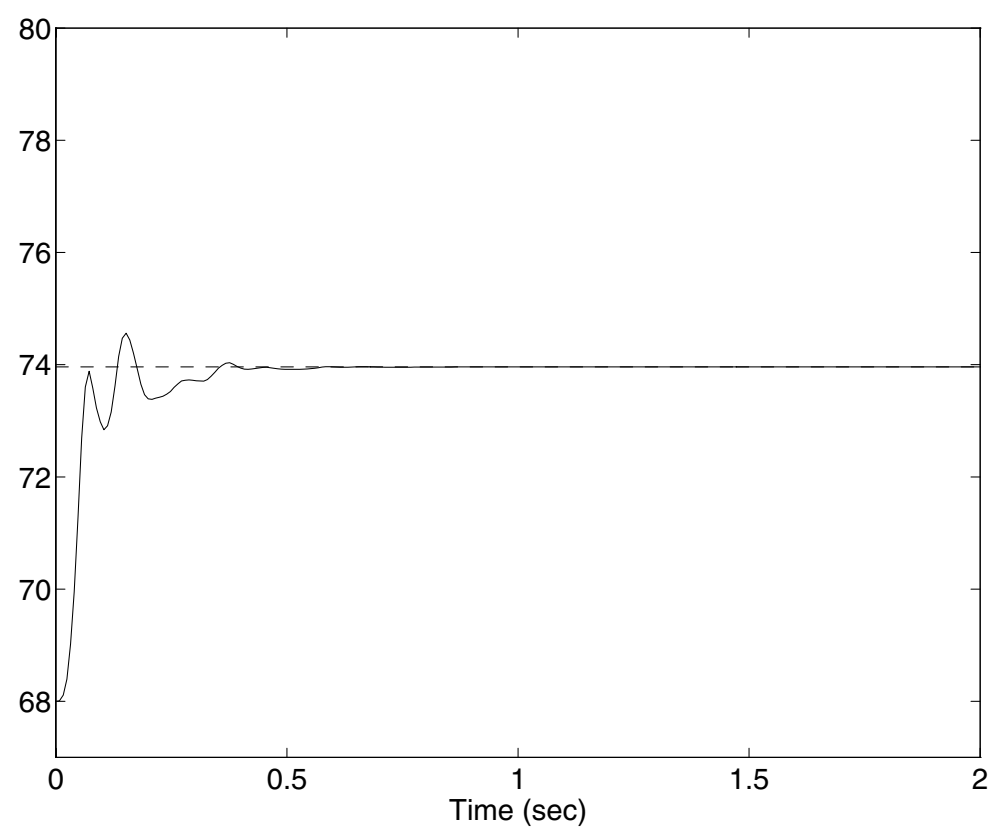

Figure 5. Actual and estimated values of $E I$ - no damping.

\section{Conclusions}

In this paper, we have extended and modified previous adaptive estimation schemes for hyperbolic systems that have non-symmetric, or even absent, damping bilinear form. In addition, a modification was added for systems that had slowly time varying parameters which converge to their steady state values asymptotically (or exponentially) in time. Of course, for all modifications, the adaptive estimator can identify both constant (in space) and functional parameters. The estimation of parameters in the input operator, bounded or unbounded, was also incorporated to the above modifications.

The proposed estimator, like the one given in [15], is infinite dimensional and therefore cannot be implemented. An approximation scheme was presented, which was similar to the one in [15]. Since the estimator required full plant data, a modification to the finite dimensional scheme was added to include a finite dimensional approximation to the plant data. This in a way can be viewed as an adaptive state and parameter estimator that uses input and output (through the finite dimensional approximation assumptions on the plant) information only.

A possible extension and further study to the above is the incorporation of an adaptive observer which adaptively estimates both the parameters and the state using only input and output data through a more general output operator. A possible point of reference would be the work of Lilly in [19], wherein the system is divided in two subsystems, a finite dimensional dominant system and a stable infinite dimensional residual system. Further yet, another direction towards adaptive observers would be the one used ESAIM: COCV, MAY 1998, VOL. 3, 133-162 
in [13] for a special class of infinite dimensional systems. In addition, the effect of noisy data can be taken into account by introducing robust adaptive schemes, as this was studied for the parabolic case in [17]. Further extension is the improvement of the numerical algorithms so that for fast systems the adaptive estimator can be applicable in real time. One such extension might be the incorporation of a hybrid adaptive parameter estimator in which the parameters are updated not in a single time unit but every several time units.

\section{REFERENCES}

[1] A.M. Annaswamy, K.S. Narendra: Adaptive control of a first order plant with timevarying parameter, In Proceedings of the 1989 American Control Conference, 1989, $975-980$.

[2] A.M. Annaswamy, K.S. Narendra: Adaptive control of simple time-varying systems, In Proceedings of the 28th IEEE Conference on Decision and Control, Tampa, Florida, 1989, 1014-1018.

[3] H.T. Banks, M.A. Demetriou: Adaptive parameter estimation of hyperbolic distributed parameter systems with non-symmetric damping and slowly time varying parameters: Convergence proofs and numerical results, Center for Research in Scientific Computation Report 96-31, North Carolina State University, Raleigh, 1996.

[4] H.T. Banks, M.A. Demetriou, R.C. Smith: Adaptive parameter estimation in a 2d structural acoustic model with piezoceramic actuator, In Proceedings of the 1994 American Control Conference, Baltimore, Maryland, 1994.

[5] H.T. Banks, W. Fang, R.J. Silcox, R.C. Smith: Approximation methods for control of structural acoustics models with piezoceramic actuators, Journal of Intelligent Material Systems and Structures, 4, 1993, 98-116.

[6] H.T. Banks, K. Ito: A unified framework for approximation and inverse problems for distributed parameter systems, Control-Theory and Advanced Technology, 4, 1988, $73-90$.

[7] H.T. Banks, K. Ito, Y. Wang: Well posedness for damped second order systems with unbounded input operators, Differential and Integral Equations, 8(3), 1995, 587-606.

[8] H.T. Banks, R.J. Silcox, R.C. Smith: The modeling and control of acoustic/structure interaction problems via piezoceramic actuators: 2-d numerical examples, ASME Journal of Vibration and Acoustics, 116, 1994, 386-396.

[9] H.T. Banks, R.C. Smith: Feedback control of noise in a 2-d nonlinear structural acoustics model, Continuous and Discrete Dynamical Systems, 1, 1995, 119-149.

[10] H.T. Banks, R.C. Smith, Y. Wang: Smart Material Structures: Modeling, Estimation and Control, Wiley-Masson, New York, 1996.

[11] H.T. Banks, Y. Wang, D.J. Inman, J.C. Slater: Approximation and parameter identification for damped second order systems with unbounded input operators, Control: Theory and Advanced Technology, 10, 1994, 873-892.

[12] J. Baumeister, W. Scondo, M.A. Demetriou, I.G. Rosen: On-line parameter estimation for infinite dimensional dynamical systems, SIAM J. Control and Optimization, 35(2), 1997, 678-713.

[13] R.F. Curtain, M.A. Demetriou, K. Ito: Adaptive observers for structurally perturbed infinite dimensional systems, In Proceedings of the 36th IEEE Conference on Decision and Control, San Diego, California, 1997.

[14] M.A. Demetriou: Adaptive Parameter Estimation of Abstract Parabolic and Hyperbolic Distributed Parameter Systems, PhD thesis, Department of Electrical Engineering - Systems, University of Southern California, Los Angeles, 1993.

[15] M.A. Demetriou, I.G. Rosen: Adaptive identification of second order distributed parameter systems, Inverse Problems, 10(2), 1994, 261-294.

[16] M.A. Demetriou, I.G. Rosen: On the persistence of excitation in the adaptive identification of distributed parameter systems, IEEE Trans. on Automatic Control, 39(5), 1994, 1117-1123. 
[17] M.A. Demetriou, I.G. Rosen: Robust adaptive estimation schemes for parabolic distributed parameter systems, In Proceedings of the 36th IEEE Conference on Decision and Control, San Diego, California, 1997.

[18] J.K. Hale: Ordinary Differential Equations, Wiley-Interscience, John Wiley and Sons, New York, 1969.

[19] J.H. Lilly: Finite dimensional adaptive observers applied to distributed parameter systems, IEEE Trans. on Automatic Control, 38(3), 1993, 469-474.

[20] J.L. Lions: Optimal Control of Systems Governed by Partial Differential Equations, Springer-Verlag, New York, 1971.

[21] J.L. Lions, E. Magenes: Non-Homogeneous Boundary Value Problems, I, SpringerVerlag, New York, 1972.

[22] K.S. Narendra, A.M. Annaswamy: Stable Adaptive Systems, Prentice Hall, Englewood Cliffs, 1989.

[23] A. Pazy: Semigroups of Linear Operators and Applications to Partial Differential Equations, Springer-Verlag, New York, 1983.

[24] I.G. Rosen, M.A. Demetriou: Adaptive estimation of a flexible beam, In Proceedings of the 1993 IEEE Regional Conference on Aerospace Control Systems, Westlake Village, California, 1993.

[25] W. Scondo: Ein Modellabgleichsverfahren zur adaptiven Parameteridentifikation in Evolutionsgleichungen, $\mathrm{PhD}$ thesis, Johann Wolfgang Goethe-Universitat zu Frankfurt am Main, Frankfurt am Main, Germany, 1987.

[26] R.E. Showalter: Hilbert Space Methods for Partial Differential Equations, Pitman, London, 1977.

[27] H. Tanabe: Equations of Evolution, Pitman, London, 1979.

[28] J. Wloka: Partial differential equations, Cambridge University Press, Cambridge, 1987. 